\title{
Review Article \\ Selected Experimental Results from Heavy-Ion Collisions at LHC
}

\author{
Ranbir Singh, ${ }^{1}$ Lokesh Kumar, ${ }^{2,3}$ Pawan Kumar Netrakanti, ${ }^{4}$ and Bedangadas Mohanty ${ }^{3}$ \\ ${ }^{1}$ Physics Department, University of Jammu, Jammu 180001, India \\ ${ }^{2}$ Kent State University, Kent, OH 44242, USA \\ ${ }^{3}$ School of Physical Sciences, National Institute of Science Education and Research, Bhubaneswar 751005, India \\ ${ }^{4}$ Nuclear Physics Division, Bhabha Atomic Research Centre, Mumbai 400085, India
}

Correspondence should be addressed to Bedangadas Mohanty; bedanga@niser.ac.in

Received 10 April 2013; Revised 29 July 2013; Accepted 6 August 2013

Academic Editor: Edward Sarkisyan-Grinbaum

Copyright (C) 2013 Ranbir Singh et al. This is an open access article distributed under the Creative Commons Attribution License, which permits unrestricted use, distribution, and reproduction in any medium, provided the original work is properly cited.

We review a subset of experimental results from the heavy-ion collisions at the Large Hadron Collider (LHC) facility at CERN. Excellent consistency is observed across all the experiments at the LHC (at center of mass energy $\sqrt{s_{\mathrm{NN}}}=2.76 \mathrm{TeV}$ ) for the measurements such as charged particle multiplicity density, azimuthal anisotropy coefficients, and nuclear modification factor of charged hadrons. Comparison to similar measurements from the Relativistic Heavy Ion Collider (RHIC) at lower energy $\left(\sqrt{s_{\mathrm{NN}}}=200 \mathrm{GeV}\right)$ suggests that the system formed at LHC has a higher energy density and larger system size and lives for a longer time. These measurements are compared to model calculations to obtain physical insights on the properties of matter created at the RHIC and LHC.

\section{Introduction}

The main goal of the high energy heavy-ion collisions is to study the phase structure of the quantum chromodynamic (QCD) phase diagram [1-3]. One of the most interesting aspects of these collisions is the possibility of forming a phase of deconfined quarks and gluons, a system that is believed to have existed in a few microseconds-old universe. First principle QCD calculations suggest that it is possible to have such a state of matter if the temperatures attained can be of the order of the QCD scale ( 200 MeV) [4-6]. In laboratory, such temperatures could be attained by colliding heavy ions at relativistic energies. Furthermore, in very high energy collisions of heavy ions at the LHC and RHIC, the lifetime of the deconfined phase may be long enough to allow for the detailed study of the fundamental constituents (quarks and gluons) of the visible matter.

The results from heavy-ion collisions at RHIC have clearly demonstrated the formation of a deconfined system of quarks and gluons in $\mathrm{Au}+\mathrm{Au}$ collisions at $\sqrt{s_{\mathrm{NN}}}=200 \mathrm{GeV}$ [7-11]. The produced system exhibits copious production of strange hadrons, shows substantial collectivity developed in the partonic phase, and exhibits suppression in high transverse momentum $\left(p_{T}\right)$ hadron production relative to $p+p$ collisions and small fluidity as reflected by a small value of viscosity to entropy density ratio $(\eta / s)$. A factor of 14 increase in $\sqrt{s_{\mathrm{NN}}}$ for $\mathrm{Pb}+\mathrm{Pb}$ collisions at LHC is expected to unravel the temperature dependence of various observables and to extend the kinematic reach in rapidity and $p_{T}$ of previous measurements at RHIC. On the other hand, the beam energy scan program at RHIC is expected to provide additional details of the QCD phase diagram not accessible at the LHC [12].

In this review paper, we discuss a subset of results that have come out from LHC Pb $+\mathrm{Pb}$ collisions at $\sqrt{s_{\mathrm{NN}}}=2.76$ $\mathrm{TeV}$. We have divided the discussion into three sections. In the second section, we discuss the consistency of various measurements among the three LHC experiments that have heavy-ion programs: ALICE, ATLAS, and CMS. Section 2.1 discusses the results on the charged particle multiplicity. Section 2.2 discusses the results on azimuthal anisotropy, and Section 2.3 discusses the results on the nuclear modification factor.

In the third section, we make a comparative study between similar observables measured at lower energy collisions at RHIC and those from LHC. In doing this, we 


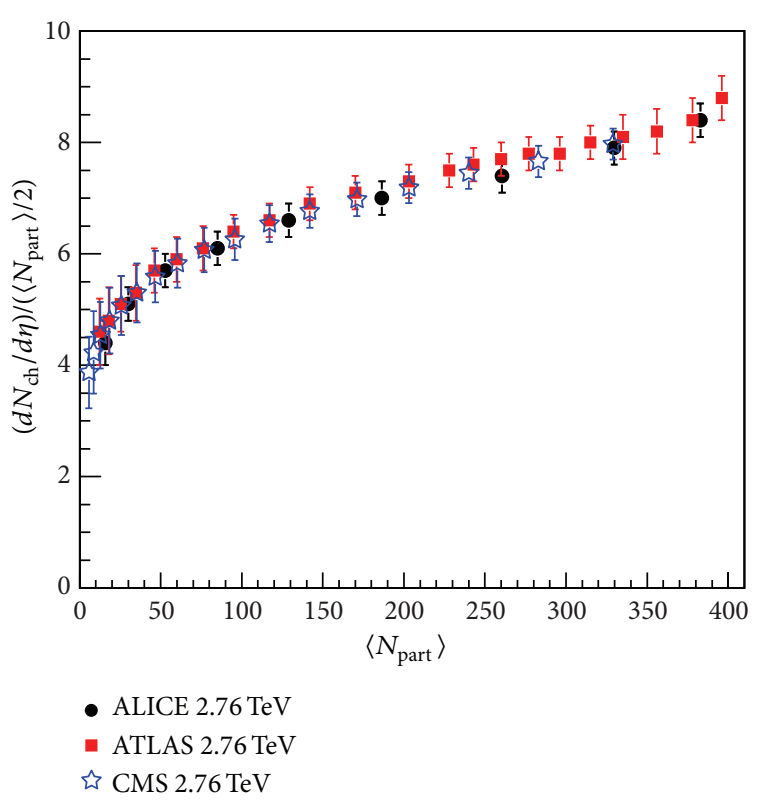

FIgURE 1: (Color online) Average charged particle multiplicity per unit pseudorapidity $\left(d N_{\mathrm{ch}} / d \eta\right)$ at midrapidity per participating nucleon $\left(\left\langle N_{\text {part }}\right\rangle\right)$ pair plotted as a function of $\left\langle N_{\text {part }}\right\rangle$ for $\mathrm{Pb}+\mathrm{Pb}$ collisions at $\sqrt{s_{\mathrm{NN}}}=2.76 \mathrm{TeV}$. The measurements are shown from ALICE [14], CMS [15], and ATLAS [16] experiments.

highlight the additional information that heavy-ion collisions at LHC bring compared to RHIC. In Section 3.1, we discuss the bulk properties at freeze-out that include results on multiplicity, average transverse mass and Bjorken energy density, volume and decoupling time, kinetic freeze-out temperature and average flow velocity, and fluctuations. Section 3.2 is devoted on the results to azimuthal anisotropy, where we discuss the energy dependence of $p_{T}$ integrated $v_{2}$, dependence of various azimuthal anisotropy coefficients on $p_{T}$, and flow fluctuations. In Section 3.3, we discuss results for nuclear modification factor.

In the fourth section, we present a comparison of various model calculations to the corresponding measurements at LHC. We concentrate mainly on the results for charged particle multiplicity density and $K / \pi$ ratio in Section 4.1 , azimuthal anisotropy in Section 4.2, and nuclear modification factor in Section 4.3.

Finally, we summarize our observations in the last section of the paper.

\section{Consistency of Results among LHC Experiments}

2.1. Charged Particle Multiplicity. One of the first measurements to come out of the heavy-ion collision program at LHC is the charged particle multiplicity per unit pseudorapidity in $\mathrm{Pb}+\mathrm{Pb}$ collisions at $\sqrt{s_{\mathrm{NN}}}=2.76 \mathrm{TeV}$. Figure 1 shows the centrality (reflected by the number of participating nucleons, $N_{\text {part }}$, obtained from a Glauber model calculation [13]) dependence of $d N_{\mathrm{ch}} / d \eta$ at midrapidity for $\mathrm{Pb}+\mathrm{Pb}$ collisions at $\sqrt{s_{\mathrm{NN}}}=2.76 \mathrm{TeV}$ from ALICE [14], CMS [15], and ATLAS [16] experiments. The error bars reflect statistical uncertainties. The ATLAS measurements of $d N_{\mathrm{ch}} /\left.d \eta\right|_{\eta=0}$ are obtained over $|\eta|<0.5$ using a minimum bias trigger with a central solenoid magnet off data set. The charged particles are reconstructed using two different algorithms using the information from pixel detectors covering $|\eta|<2$.0. The $N_{\text {part }}$ values are obtained by comparing the summed transverse energy in the forward calorimeter over a pseudorapidity range $3.2<|\eta|<4.9$ to a Glauber model simulation. The CMS results for $d N_{\mathrm{ch}} /\left.d \eta\right|_{\eta=0}$ are from the barrel section of the pixel tracker covering $|\eta|<2.5$. The minimum bias trigger data set was in the magnetic field off configuration so as to improve the acceptance of low $p_{T}$ particles. The centrality determinations as in the case of ATLAS experiment are done using information from hadron forward calorimeter $(2.9<|\eta|<5.2)$ and Glauber model simulations. The ALICE measurement uses a minimum bias data set from the silicon pixel detector $(|\eta|<2.0)$. The centrality selection is carried out using signals from VZERO detectors (2 arrays of 32 scintillator tiles) covering the regions $2.8<\eta<5.1$ and $-3.7<$ $\eta<-1.7$, along with the corresponding Glauber modeling of the data.

In spite of the difference in operating conditions and measurement techniques, the $d N_{\mathrm{ch}} / d \eta$ versus $N_{\text {part }}$ results for $\mathrm{Pb}+\mathrm{Pb}$ collisions at $\sqrt{s_{\mathrm{NN}}}=2.76 \mathrm{TeV}$ show a remarkable consistency across the three experiments. The results show that the charged particle multiplicity per unit pseudorapidity per nucleon pair increases from peripheral to central collisions. This gradual increase in $d N_{\mathrm{ch}} / d \eta$ per participating nucleon pair indicates that in central head-on collisions, where the number of participating nucleons is more, the charged particle production is different compared to that in peripheral collisions.

2.2. Azimuthal Anisotropy. Azimuthal anisotropy has been studied in great detail in heavy-ion collision experiments. It can provide information about initial stages of heavyion collisions. Figure 2 (top panels) shows the azimuthal anisotropy of produced charged particles $\left(v_{n}=\langle\cos (n(\phi-\right.$ $\left.\left.\left.\Psi_{n}\right)\right)\right\rangle$ ) as a function of $p_{T}$ for $30-40 \% \mathrm{~Pb}+\mathrm{Pb}$ collisions at $\sqrt{s_{\mathrm{NN}}}=2.76 \mathrm{TeV}$ from the three different experiments: ATLAS, ALICE, and CMS. Here, $\phi$ is the azimuthal angle of the produced particles, and $\Psi_{n}$ is the $n$th order reaction plane angle measured in the experiments. The left panel in the figure corresponds to $v_{2}$, the middle panel corresponds to $v_{3}$, and the right panel corresponds to $v_{4}$, respectively. Bottom panels show the ratio of the experimental data to a polynomial fit to the ALICE data.

In the CMS experiment [17-20], the $v_{2}$ measurements use the information from the silicon tracker in the region $|\eta|<2.5$ with a track momentum resolution of $1 \%$ at $p_{T}=$ $100 \mathrm{GeV} / \mathrm{c}$ kept within a magnetic field of 3.8 Tesla. The event plane angle $\left(\Psi_{2}\right)$ is obtained using the information on the energy deposited in the hadron forward calorimeter. A minimum $\eta$ gap of 3 units is kept between the particles used for obtaining $\Psi_{2}$ and $v_{2}$. This ensures suppression of nonflow correlations which could arise, for example, from dijets. The event plane resolution obtained using three subevents technique varies from 0.55 to 0.84 , depending on the collision 


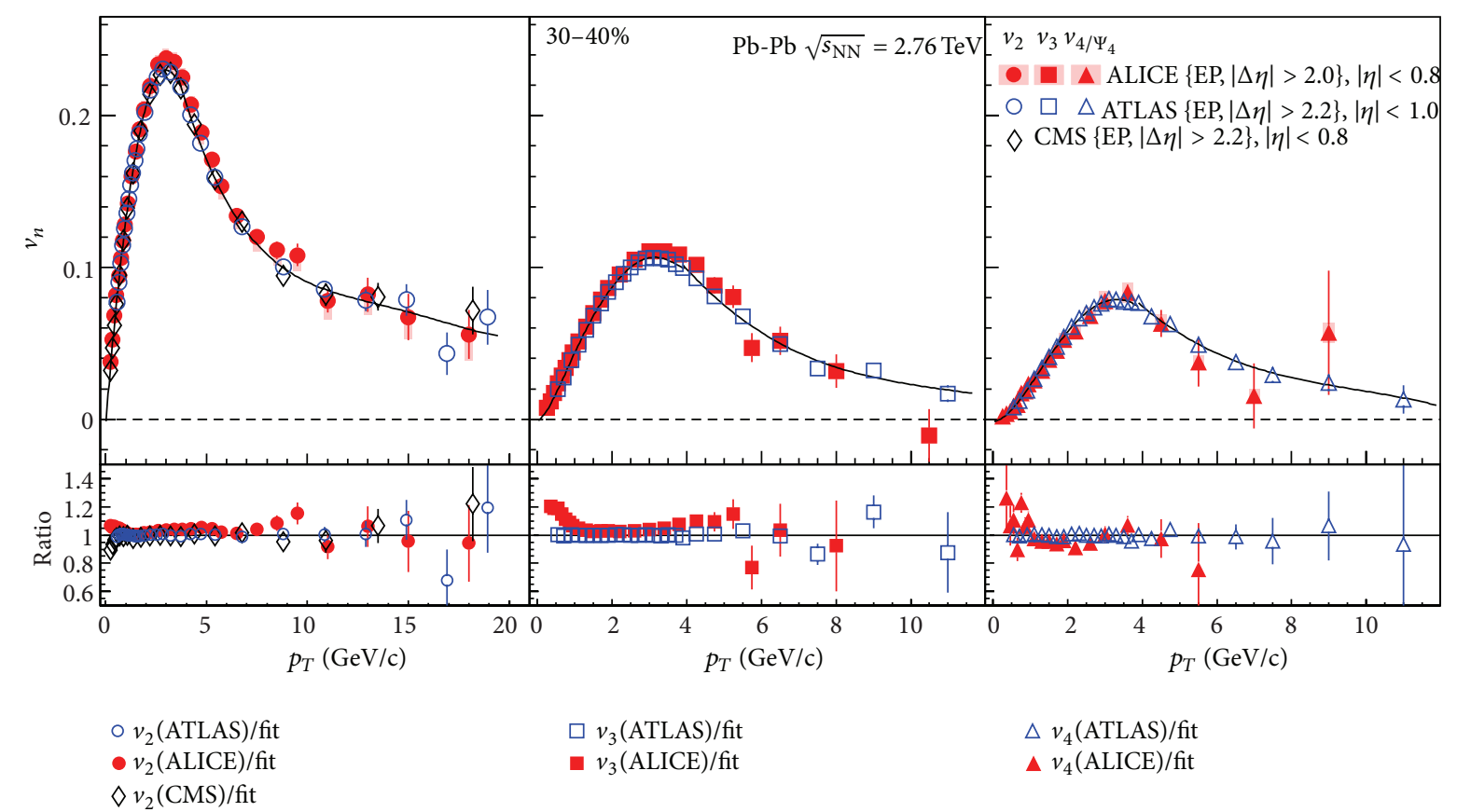

Figure 2: (Color online) $v_{n}$ versus $p_{T}$ at midrapidity for $30-40 \% \mathrm{~Pb}+\mathrm{Pb}$ collisions at $\sqrt{s_{\mathrm{NN}}}=2.76 \mathrm{TeV}$. The results are shown from different LHC experiments: CMS [17-20], ATLAS [21-24], and ALICE [25]. The bottom panels show the ratio of the experimental data to a polynomial fit to the ALICE data.

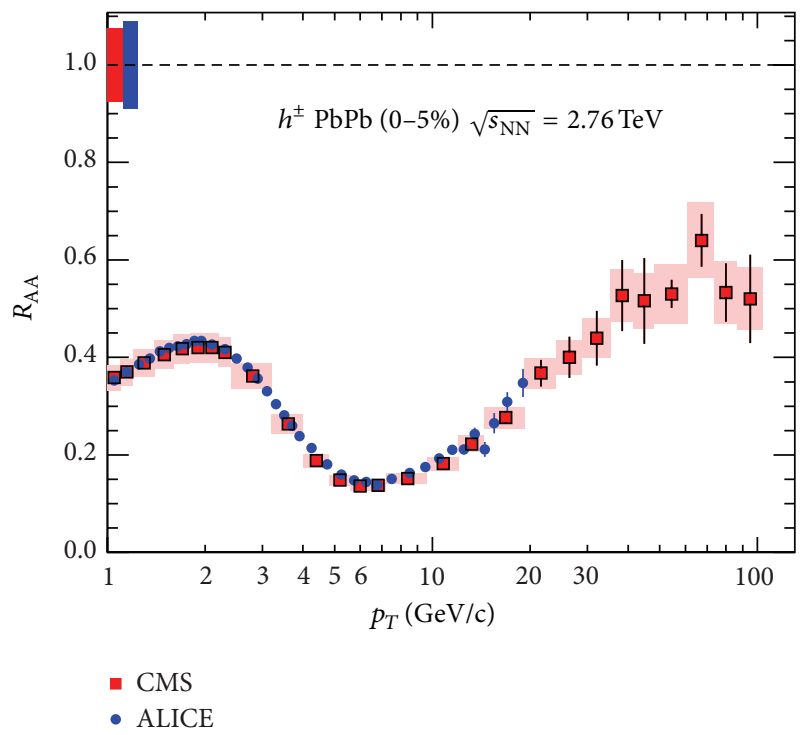

Figure 3: (Color online) Nuclear modification factor $R_{\mathrm{AA}}$ of charged hadrons measured by ALICE [26] and CMS [27] experiments at midrapidity for $0-5 \%$ most central $\mathrm{Pb}-\mathrm{Pb}$ collisions at $\sqrt{s_{\mathrm{NN}}}=$ $2.76 \mathrm{TeV}$. The boxes around the data denote $p_{T}$-dependent systematic uncertainties. The systematic uncertainties on the normalization are shown as boxes at $R_{\mathrm{AA}}=1$.

centrality. The ATLAS experiment [21-24] measured $v_{n}$ using the inner detectors in the $|\eta|<2.5$, kept inside a 2 Tesla field of superconducting solenoid magnet. The event planes are obtained using forward calorimeter information, with a resolution varying from 0.2 to 0.85 , depending on collision centrality. The ALICE experiment [25] measured $v_{n}$ using charged tracks reconstructed from the Time Projection Chamber $(|\eta|<0.8)$; the event plane was obtained using information from VZERO detectors kept at a large rapidity gap from the TPC. The momentum resolution of the tracks is better than $5 \%$.

A very nice agreement for $v_{2}, v_{3}$, and $v_{4}$ versus $p_{T}$ is found between all the experiments to a level of within $10 \%$ for most of the $p_{T}$ ranges presented. The results show an increase of $v_{2}$, $v_{3}$, and $v_{4}$ values with $p_{T}$ for the low $p_{T}$ and a decrease for $p_{T}$ above $\sim 3 \mathrm{GeV} / \mathrm{c}$. The hydrodynamical evolution of the system affects most of the low $p_{T}$ particles and hence the increasing $v_{n}$ at low $p_{T}$.

2.3. Nuclear Modification Factor. One of the established signatures of the QGP at top RHIC energy is the suppression of high transverse momentum $\left(p_{T}\right)$ particles in heavy-ion collisions compared to corresponding data from the binary collisions scaled $p+p$ collisions. It has been interpreted in terms of energy loss of partons in QGP. This phenomenon is referred to as the jet quenching in a dense partonic matter. The corresponding measurement is called the nuclear modification factor $\left(R_{\mathrm{AA}}\right)$.

Figure 3 shows the nuclear modification factor for inclusive charged hadrons measured at midrapidity in LHC experiments for $\mathrm{Pb}+\mathrm{Pb}$ collisions at $\sqrt{s_{\mathrm{NN}}}=2.76 \mathrm{TeV}$. The nuclear modification factor is defined as $R_{\mathrm{AA}}=$ $\left(d N_{\mathrm{AA}} / d \eta d^{2} p_{T}\right) /\left(T_{\mathrm{AB}} d \sigma_{\mathrm{NN}} / d \eta d^{2} p_{T}\right)$. Here, the overlap integral $T_{\mathrm{AB}}=N_{\text {binary }} / \sigma_{\text {inelastic }}^{p p}$ with $N_{\text {binary }}$ being the number of binary collisions commonly estimated from Glauber model 
calculation and $d \sigma_{\mathrm{NN}} / d \eta d^{2} p_{T}$ is the cross section of charged hadron production in $p+p$ collisions at $\sqrt{s}=2.76 \mathrm{TeV}$.

The ALICE experiment [26] uses the inner tracking system (ITS) and the time projection chamber (TPC) for vertex finding and tracking in a minimum bias data set. The CMS experiment [27] reconstructs charged particles based on hits in the silicon pixel and strip detectors. In order to extend the statistical reach of the $p_{T}$ spectra in the highly prescaled minimum bias data recorded in 2011, it uses unprescaled single-jet triggers. Both experiments take the value of $\sigma_{\text {inelastic }}^{p p}=64 \pm 5 \mathrm{mb}$. The result shows that the charged particle production at high $p_{T}$ in LHC is suppressed in heavyion collisions relative to nucleon-nucleon collisions. The suppression value reaches to a minimum at $p_{T} 6-7 \mathrm{GeV} / \mathrm{c}$ and then gradually increases to attain an almost constant value at $\sim 40 \mathrm{GeV} / \mathrm{c}$. This can be understood in terms of energy loss mechanism differences in intermediate and higher $p_{T}$ regions. The rise in the $R_{\mathrm{AA}}$ above $p_{T} 6-7 \mathrm{GeV} / \mathrm{c}$ may imply the dominance of the constant fractional energy loss which is the consequence of flattening of the unquenched nucleonnucleon spectrum. An excellent agreement for $R_{\mathrm{AA}}$ versus $p_{T}$ for charged hadrons in $0-5 \%$ central $\mathrm{Pb}+\mathrm{Pb}$ collisions at $\sqrt{s_{\mathrm{NN}}}=2.76 \mathrm{TeV}$ is observed between the two experiments.

Having discussed the consistency of these first measurements in $\mathrm{Pb}+\mathrm{Pb}$ collisions among different experiments, the major detectors used, acceptances, and ways to determine centrality and event plane, we now discuss the comparison between measurements at RHIC and LHC heavy-ion collisions.

\section{Comparison of LHC and RHIC Results}

In the first subsection, we discuss the energy dependence of basic measurements made in heavy-ion collisions. These include $d N_{\mathrm{ch}} / d \eta,\left\langle m_{T}\right\rangle\left(m_{T}=\sqrt{p_{T}^{2}+m^{2}}\right.$; here, $m$ represents mass of hadron), Bjorken energy density $\left(\epsilon_{\mathrm{Bj}}\right)$, life time of the hadronic phase $\left(\tau_{f}\right)$, system volume at the freeze-out, kinetic and chemical freeze-out conditions, and finally, the fluctuations in net-charge distributions. In the next subsection, we discuss the energy dependence of $p_{T}$ integrated $v_{2}$, $v_{n}$ versus $p_{T}$, and flow fluctuations at RHIC and LHC. In the final subsection, we compare the nuclear modification factor for hadrons produced in heavy-ion collisions at RHIC and LHC.

\subsection{Bulk Properties at Freeze-Out}

3.1.1. Multiplicity. Figure 4(a) shows the charged particle multiplicity density at midrapidity $\left(d N_{c h} / d \eta\right)$ per participating nucleon pair produced in central heavy-ion collisions versus $\sqrt{s_{\mathrm{NN}}}$. We observe that the charged particle production increases by a factor 2 as the energy increases from RHIC to LHC. The energy dependence seems to rule out a logarithmic dependence of particle production with $\sqrt{s_{\mathrm{NN}}}$ and supports a power law type of dependence on $\sqrt{s_{\mathrm{NN}}}$. The red solid curve seems to describe the full energy range. More detailed discussions on the energy dependence of these measurements can be found in [28].
Figure 4(b) shows the excess of $d N_{\text {ch }} / d \eta /\left\langle N_{\text {part }}\right\rangle$ in $\mathrm{A}+\mathrm{A}$ collisions $[15,16,29-37]$ over corresponding yields in $p+p(\bar{p})$ [38-47] and $p(d)+$ A collisions $[29,48,49]$. This observation also seen at RHIC persists at LHC but is proportionately larger at the higher energy collisions at the LHC. A power law fit to the $p+p$ collision charged particle multiplicity density leads to a dependence $\sim s^{0.11}$, while those for A + A collisions go as $\sim s^{0.15}$. There is no scaling observed in the charged particle multiplicity density per participating nucleon, when compared between elementary collisions like $p+p$ and heavyion collisions. This is a clear indication that $\mathrm{A}+\mathrm{A}$ collisions at RHIC and LHC are not a simple superposition of several $p+p$ collisions, whereas the $p+$ A collisions scale with the $p+p$ collisions.

3.1.2. Average Transverse Mass and Bjorken Energy Density. Figure 5(a) shows the $\left\langle m_{T}\right\rangle$ values for pions in central heavyion collisions as a function of $\sqrt{s_{\mathrm{NN}}}$. The $\left\langle m_{T}\right\rangle$ value increases with $\sqrt{s_{\mathrm{NN}}}$ at lower AGS energies [50,51], stays independent of $\sqrt{s_{\mathrm{NN}}}$ for the SPS energies $[52,53]$, and then tends to rise further with increasing $\sqrt{s_{\mathrm{NN}}}$ at the higher beam energies of LHC. About 25\% increase in $\left\langle m_{T}\right\rangle$ is observed from RHIC $[41,54]$ to LHC [55]. For a thermodynamic system, $\left\langle m_{T}\right\rangle$ can be an approximate representation of the temperature of the system, and $d N / d y \propto \ln \left(\sqrt{s_{\mathrm{NN}}}\right)$ may represent its entropy [56]. In such a scenario, the observations could reflect the characteristic signature of a phase transition, as proposed by Van Hove [57]. Then, the constant value of $\left\langle m_{T}\right\rangle$ versus $\sqrt{s_{\mathrm{NN}}}$ has one possible interpretation in terms of formation of a mixed phase of a QGP and hadrons during the evolution of the heavy-ion system. The energy domains accessed at RHIC and LHC will then correspond to partonic phase, while those at AGS would reflect hadronic phase. However, there could be several other effects to which $\left\langle m_{T}\right\rangle$ is sensitive, which also need to be understood for proper interpretation of the data [56].

Figure 5(b) shows the product of the estimated Bjorken energy density $\left(\epsilon_{\mathrm{Bj}}=\left(1 /\left(A_{\perp} \tau\right)\right) d E_{T} / d y ; A_{\perp}\right.$ [58] is the transverse overlap area of the nuclei, and $E_{T}$ is the transverse energy) and formation time $(\tau)$ as a function of $\sqrt{s_{\mathrm{NN}}}$ [59$64]$. The product of energy density and the formation time at LHC seem to be a factor of 3 larger compared to those attained at RHIC. If we assume the same value of $\tau_{0}(=1 \mathrm{fm} / \mathrm{c})$ for LHC and RHIC, the Bjorken energy density is about a factor of 3 larger at the LHC compared to that at RHIC in central collisions.

3.1.3. Volume and Decoupling Time. The top panel of Figure 6 shows the energy dependence of the product of the three radii $\left(R_{\text {out }}, R_{\text {side }}\right.$, and $\left.R_{\text {long }}\right)$ obtained from pion HBT or BoseEinstein correlation analysis. Here, the "out" corresponds to the axis pointing along the pair transverse momentum, the "side" to the axis perpendicular to it in the transverse plane, and the "long" corresponds to the axis along the beam (Bertsch-Pratt convention $[65,66]$ ). The product of the radii is connected to the volume of the homogeneity region at the last interaction. The product of the three radii shows a linear dependence on the charged-particle pseudorapidity density. 


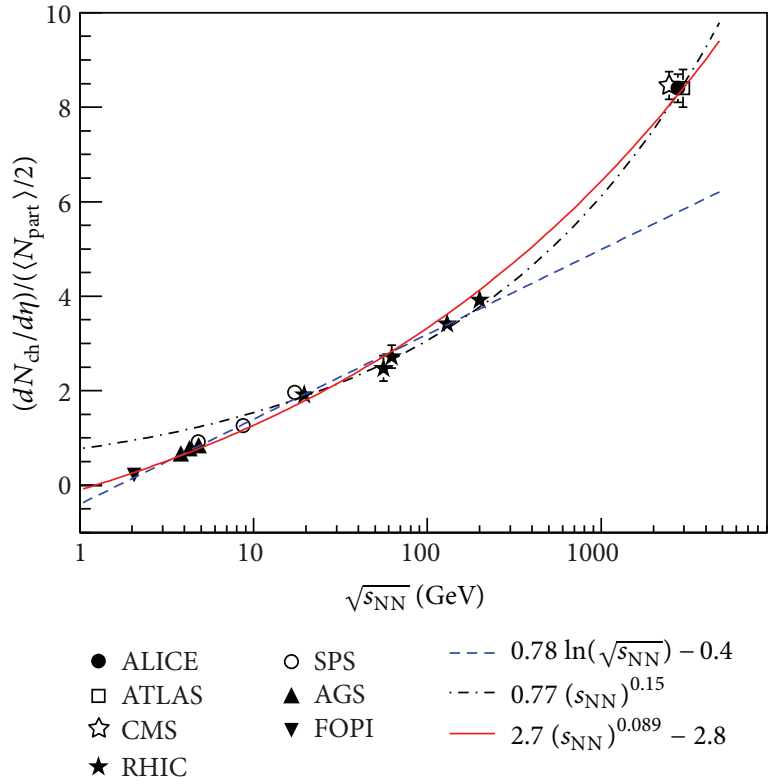

(a)

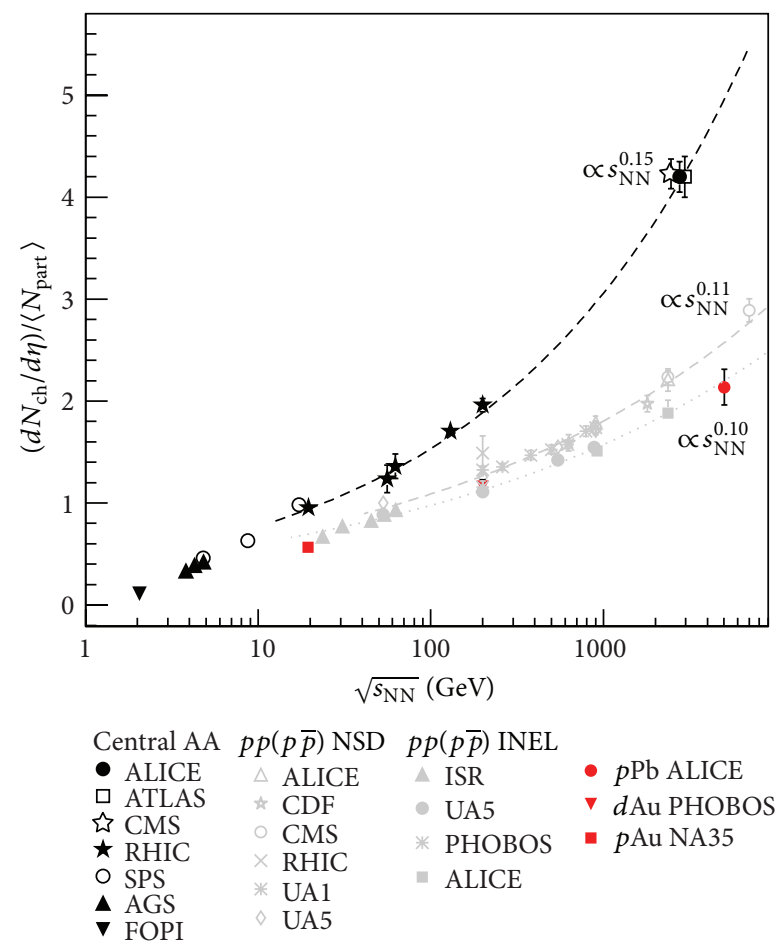

(b)

FIgURE 4: (Color online) (a) $d N_{\mathrm{ch}} / d \eta$ per participating nucleon pair at midrapidity in central heavy-ion collisions as a function of $\sqrt{s_{\mathrm{NN}}}$. (b) Comparison of $d N_{\mathrm{ch}} / d \eta$ per participating nucleon at midrapidity in central heavy-ion collisions $[15,16,29-37]$ to corresponding results from $p+p(\bar{p})[38-47]$ and $p(d)+$ A collisions $[29,48,49]$.

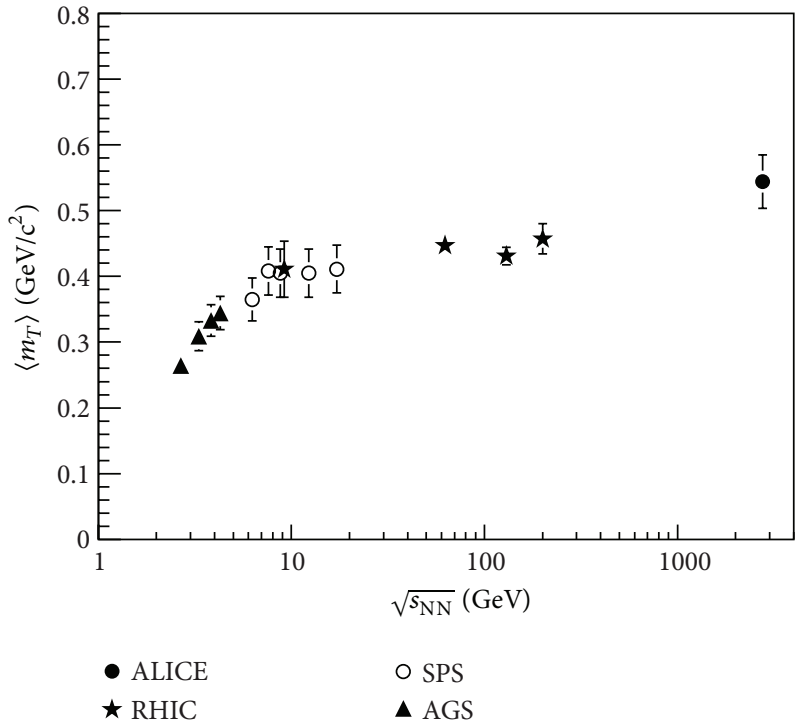

(a)

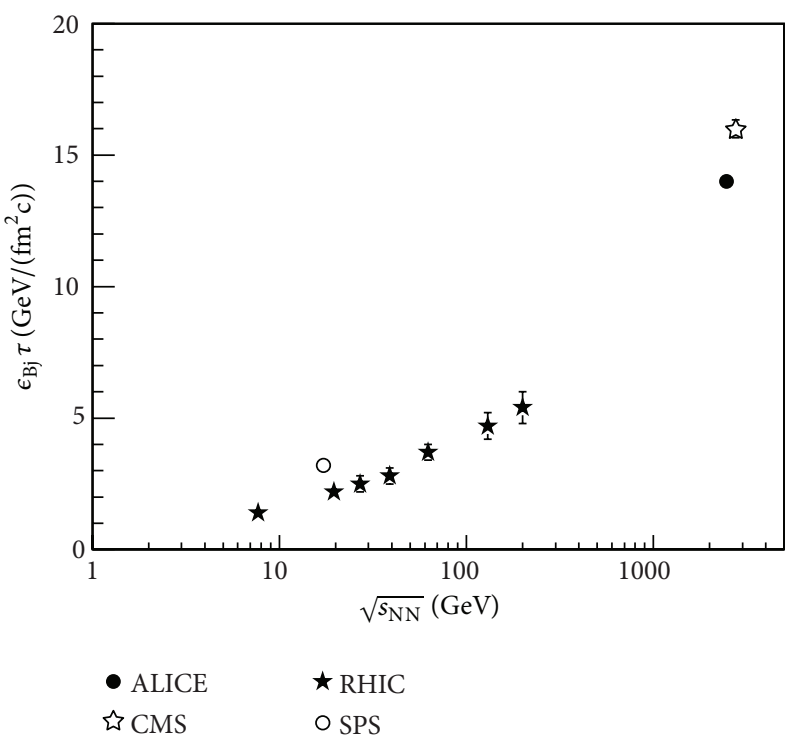

(b)

FIGURE 5: (a) $\left\langle m_{T}\right\rangle$ for charged pions in central heavy-ion collisions at midrapidity for AGS [50, 51], SPS [52, 53], RHIC [41, 54], and LHC [55] energies. The errors shown are the quadrature sum of statistical and systematic uncertainties. (b) The product of Bjorken energy density, $\epsilon_{\mathrm{Bj}}$ [58], and the formation time $(\tau)$ in central heavy-ion collisions at midrapidity as a function of $\sqrt{s_{\mathrm{NN}}}[59-64]$. 


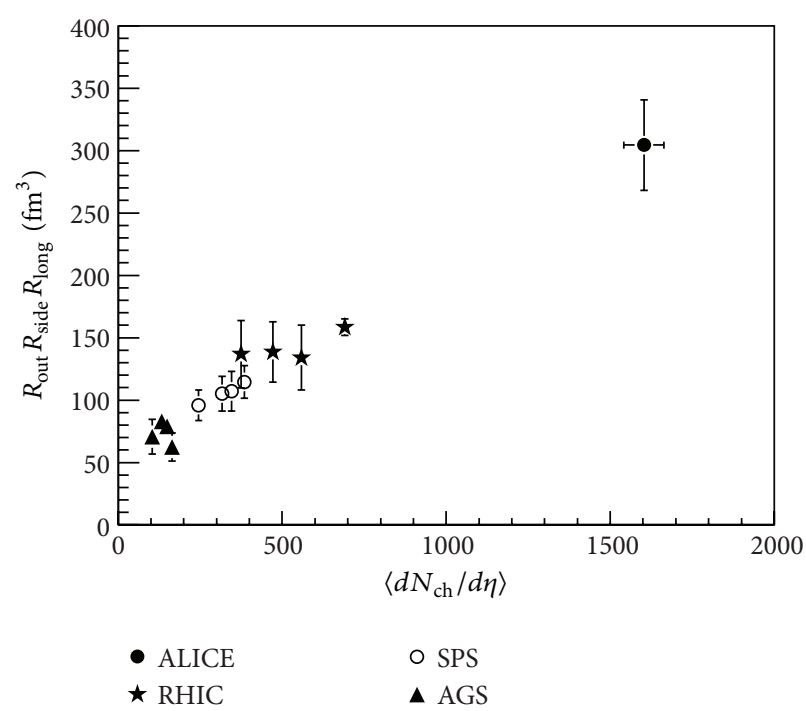

(a)

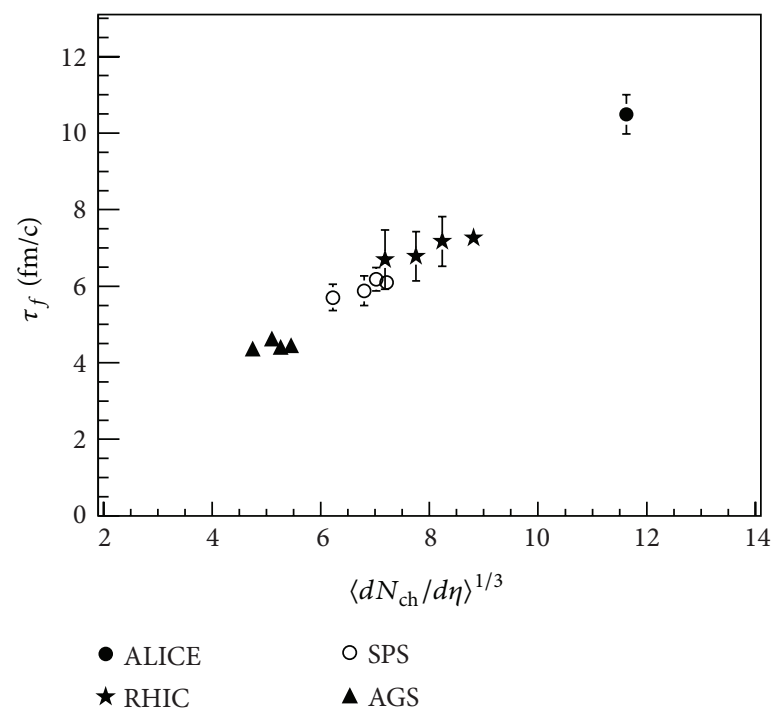

(b)

FIGURE 6: (a) Product of the three pion HBT radii at $k_{T}$ (average transverse momenta of two pions) $=0.3 \mathrm{GeV} / \mathrm{c}$ for central heavy-ion collisions at AGS [68], SPS [69, 70], RHIC [71, 72], and LHC [73] energies. (b) The decoupling time extracted from $R_{\text {long }}\left(k_{T}\right)$ for central heavy-ion collisions at midrapidity at AGS, SPS, RHIC, and LHC energies as a function of $\left(d N_{\mathrm{ch}} / d \eta\right)^{1 / 3}$.

The data indicates that the volume of homogeneity region is two times larger at the LHC than at RHIC.

Furthermore, within a hydrodynamic picture, the decoupling time for hadrons $\left(\tau_{f}\right)$ at midrapidity can be estimated from the magnitude of radii $R_{\text {long }}$ as follows: $R_{\text {long }}^{2}=$ $\tau_{f}^{2} T K_{2}\left(m_{T} / T\right) / m_{T} K_{1}\left(m_{T} / T\right)$, with $m_{T}=\sqrt{m_{\pi}^{2}+k_{T}^{2}}$, where $m_{\pi}$ is the mass of the pion, $T$ is the kinetic freeze-out temperature, and $K_{1}$ and $K_{2}$ are the integer-order modified Bessel functions [67]. For the estimation of $\tau_{f}$, the average value of the kinetic freeze-out temperature $T$ is taken to be $120 \mathrm{MeV}$ from AGS to LHC energies. However, the energy dependence of kinetic freeze-out temperature, as discussed in the next subsection, would provide a more accurate description of the $\tau_{f}$ values. The extracted $\tau_{f}$ values for central heavy-ion collisions at midrapidity at AGS [68], SPS [69, 70], RHIC [71, 72], and LHC [73] energies are shown as a function of cube root of $d N_{\mathrm{ch}} / d \eta$ in the bottom panel of Figure 6. We observe that $\tau_{f}$ scales linearly with $\left(d N_{\mathrm{ch}} / d \eta\right)^{1 / 3}$ and is about $10 \mathrm{fm} / \mathrm{c}$ at LHC energies. This value is about $40 \%$ larger than at RHIC. It may be noted that the above expression ignores transverse expansion of the system and finite chemical potential for pions. Also there are uncertainties associated with freeze-out temperature that could lead to variations in the extracted $\tau_{f}$ values.

3.1.4. Freeze-Out Temperature and Radial Flow Velocity. The hadron yields and spectra reflect the properties of the bulk matter at chemical and kinetic freeze-out, respectively. Generally, the point at which the inelastic collisions cease is called the chemical freeze-out, and the point where even the elastic collisions stop is called the kinetic freeze-out.
The transverse momentum distribution of different particles contains two components: one random and the other collective. The random component can be identified with the temperature of the system at kinetic freeze-out $\left(T_{\text {kin }}\right)$. The collective component, which could arise from the matter density gradient from the center to the boundary of the fireball created in high energy nuclear collisions, is called collective flow in transverse direction $(\langle\beta\rangle)$. Using the assumption that the system attains thermal equilibrium, the blast wave formulation can be used to extract $T_{\text {kin }}$ and $\langle\beta\rangle$. These two quantities are shown in Figure 7 versus $\sqrt{s_{\mathrm{NN}}}[41,55,74-77]$. For beam energies at AGS and above, one observes a decrease in $T_{\text {kin }}$ with $\sqrt{s_{\mathrm{NN}}}$. This indicates that the higher the beam energy is, the longer interactions are among the constituents of the expanding system and the lower the temperature. From RHIC top energy to LHC, there seems to be, however, a saturation in the value of $T_{\mathrm{kin}}$. In contrast to the temperature, the collective flow increases with the increase in beam energy, rapidly, reaching a value close to 0.6 times the speed of light at the LHC energy.

Figure 8 shows the chemical freeze-out temperature $\left(T_{\mathrm{ch}}\right)$ versus the baryon chemical potential $\left(\mu_{B}\right)$ in central heavyion collisions $[41,55,78-85]$. These quantities are obtained by fitting the particle yields to a statistical model assuming thermal equilibrium within the framework of a Grand Canonical ensemble. There are two values of temperature quoted for LHC energies. A $T_{\mathrm{ch}}$ value of about $164 \mathrm{MeV}$ and fixed $\mu_{B}$ value of $1 \mathrm{MeV}$ seem to reproduce the multistrange ratios (involving $\Xi$ and $\Omega$ ) quite well but were observed to miss the data for $p / \pi$ and $\Lambda / \pi$. On the other hand, the statistical thermal model prediction with $T_{\mathrm{ch}}=152 \mathrm{MeV}$ and fixed $\mu_{B}=$ $1 \mathrm{MeV}$ fits the measured $p / \pi$ and $\Lambda / \pi$ ratios better but misses 


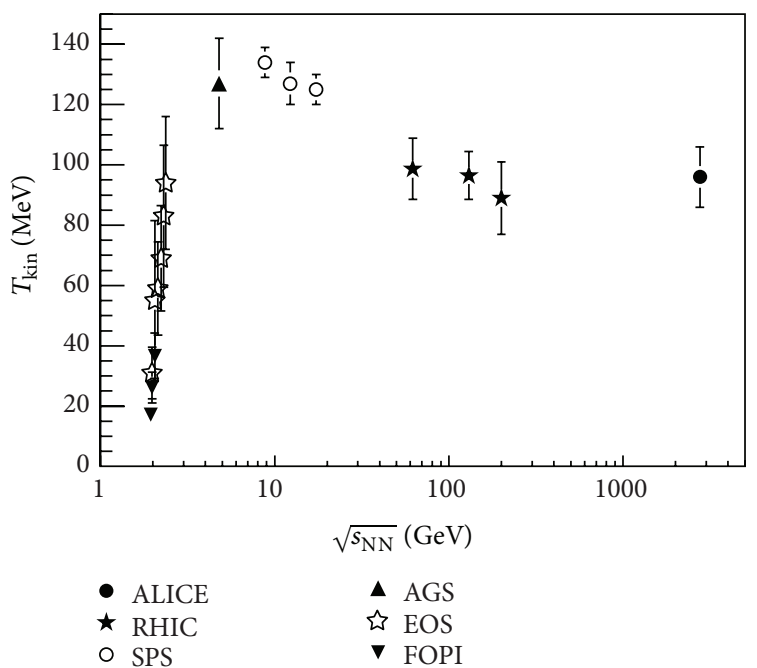

(a)

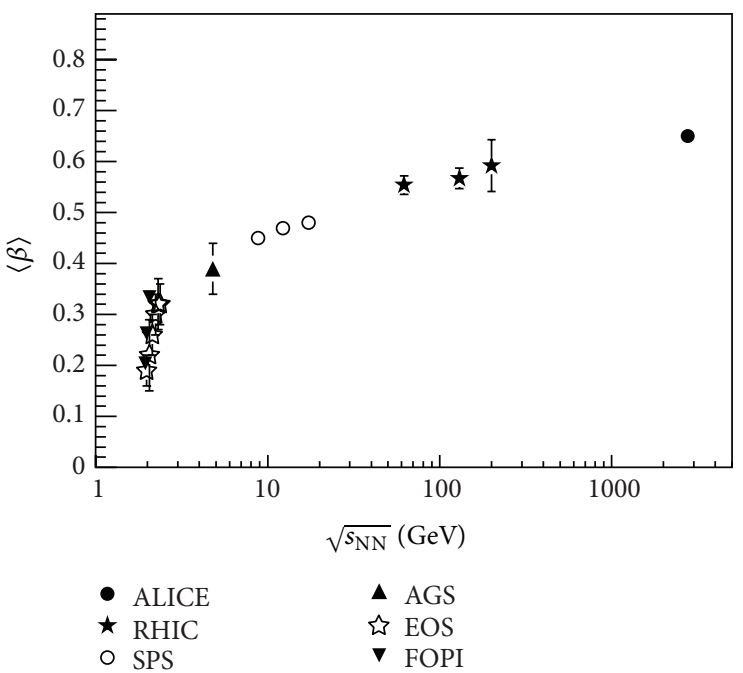

(b)

FIGURE 7: Kinetic freeze-out temperature (a) and radial flow velocity (b) in central heavy-ion collisions as a function of collision energy $[41,55,74-77]$.

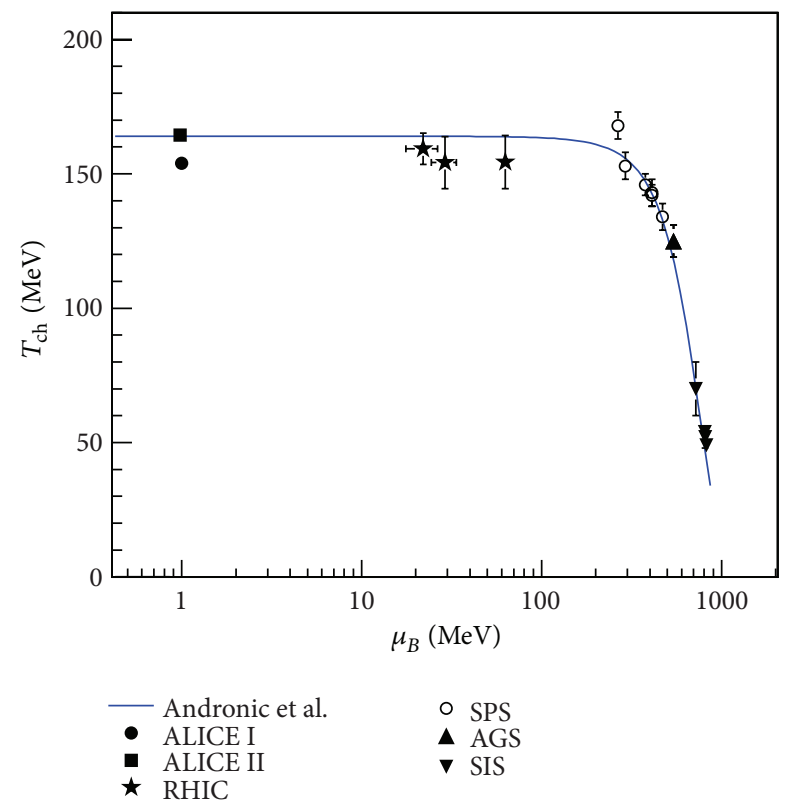

FIGURE 8: (Color online) Chemical freeze-out temperature versus baryon chemical potential in central heavy-ion collisions $[41,55,78$ 85]. The curve corresponds to model calculations from $[78,79]$.

the ratios involving multistrange hadrons [86]. This issue is not yet resolved, being possibly related to hadronic final state interactions [87]. The curve corresponds to generalization of the energy dependence of $T_{\mathrm{ch}}-\mu_{B}$ using statistical thermal model calculations $[78,79]$. The model works within the framework of a Grand Canonical ensemble and takes as input the produced particle yields from experiments to extract the freeze-out parameters such as $T_{\mathrm{ch}}$ and $\mu_{B}$.
3.1.5. Fluctuations. One of the proposed signatures to search for the phase transition from hadronic to partonic medium is to study the net-charge fluctuations in heavy-ion collisions. The partonic phase has constituents with fractional charges, while the hadronic phase has constituents with integral units of charge; hence, the measure of the fluctuations in the netcharge particle production is expected to be different in these two cases. Specifically, net-charge fluctuations are expected to be smaller if the system underwent a phase transition. However, it is important to address how these fluctuations may or may not survive the evolution of the system in the heavy-ion collisions. An experimental measure of net-charge fluctuations is defined as $\nu(+-, d y n)=\left(\left\langle N_{+}\left(N_{+}-1\right)\right\rangle /\left\langle N_{+}^{2}\right\rangle\right)+$ $\left(\left\langle N_{-}\left(N_{-}-1\right)\right\rangle /\left\langle N_{-}^{2}\right\rangle\right)-2\left(\left\langle N_{-} N_{+}\right\rangle /\left\langle N_{-}\right\rangle\left\langle N_{+}\right\rangle\right)$, where $\left\langle N_{-}\right\rangle$ and $\left\langle N_{+}\right\rangle$are average negative and positive charged particle multiplicity, respectively [88].

Figure 9 shows the product of $v(+-, d y n)$ and $\left\langle N_{\mathrm{ch}}\right\rangle$ (average number of charged particles) as a function of $\sqrt{s_{\mathrm{NN}}}$ [89-91]. We find that this observable fluctuation rapidly decreases with $\sqrt{s_{\mathrm{NN}}}$ and approaches expectation for a simple QGP-like scenario [92] as we move from RHIC to LHC energies. Given that several other observables already indicate that a hot and dense medium of color charges has been formed at RHIC and LHC, the net-charge fluctuation result may indicate that the observable $v(+-, d y n)$ is not sensitive enough to QGP physics or the process of hadronization washes out the QGP signal for this observable. It may be also noted that the model's results do not incorporate the acceptance effects and do not consider any dynamic evolution of the system like, for example, the dilution of the signals in the hadronization process.

\subsection{Azimuthal Anisotropy}

3.2.1. Energy Dependence of $p_{T}$ Integrated $v_{2}$. Figure 10 shows the $p_{T}$ integrated $v_{2}$ close to midrapidity of charged particles 


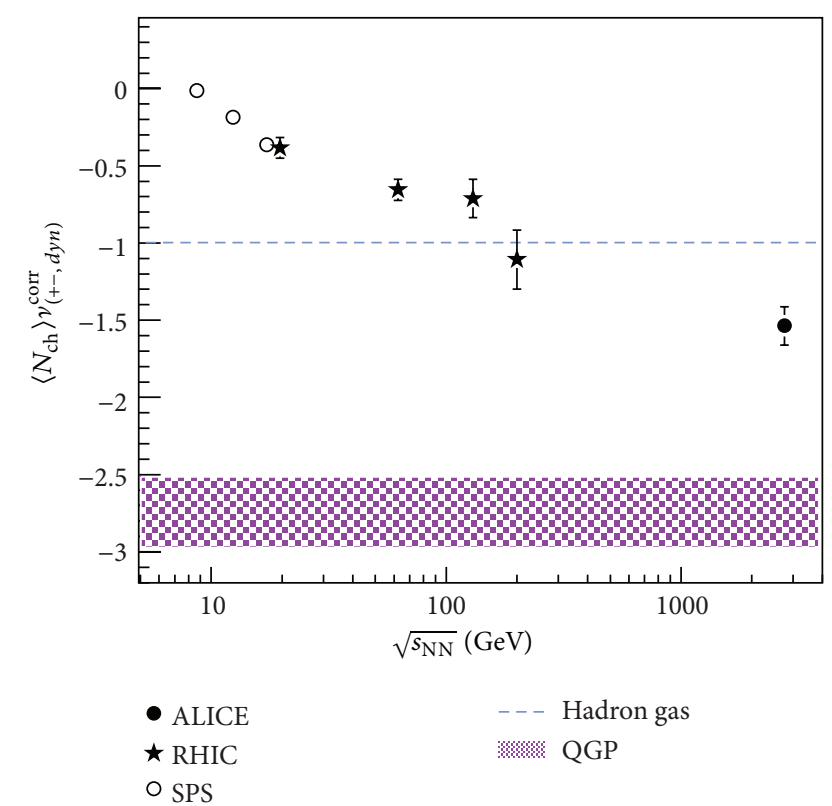

FIGURE 9: (Color online) Energy dependence of net-charge fluctuations about midrapidity in central heavy-ion collisions at SPS [89], RHIC [90], and LHC [91] energies. Also shown are the expectations from a hadron resonance gas model and for a simple QGP picture [92].

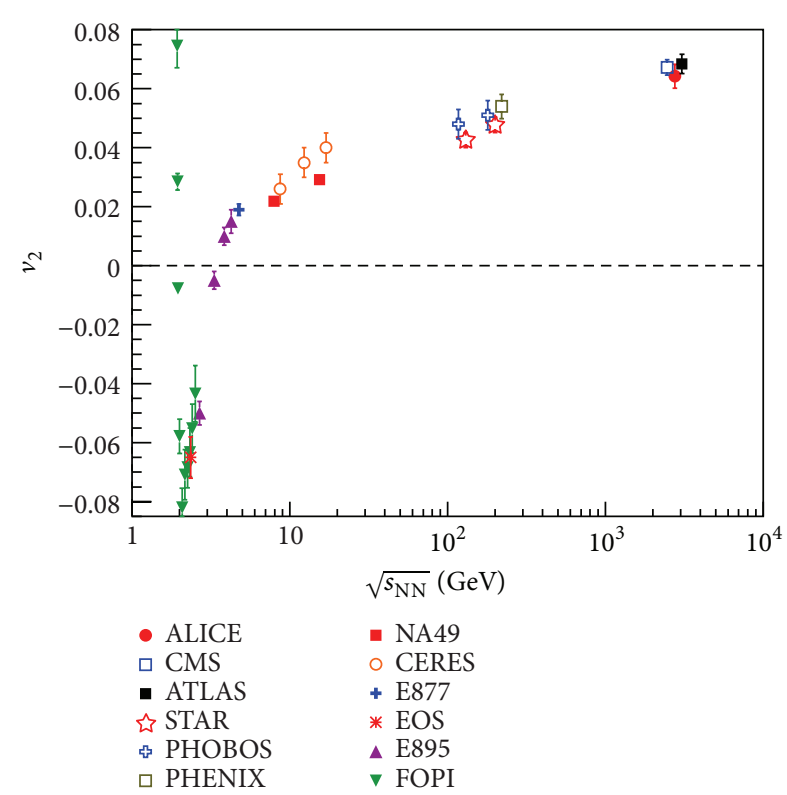

FIgURE 10: (Color online) Transverse momentum integrated $v_{2}$ close to midrapidity for charged $(Z=1)$ particles for collision centralities around $20-30 \%$ as a function of center of mass energy.

for collision centralities around $20-30 \%$ as a function of center of mass energy. We observe that there is an increase in magnitude of $v_{2}$ by about $30 \%$ from top RHIC energy $\left(\sqrt{s_{\mathrm{NN}}}=\right.$ $200 \mathrm{GeV})$ to LHC energy $\left(\sqrt{s_{\mathrm{NN}}}=2.76 \mathrm{TeV}\right)$. This needs to be viewed within the context of a similar magnitude of increase in $\left\langle p_{T}\right\rangle$ of pions from RHIC to LHC energies. The increase of $v_{2}$ beyond beam energy of $10 \mathrm{GeV}$ is logarithmic in $\sqrt{s_{\mathrm{NN}}}$. This is expected to be determined by the pressure gradient-driven expansion of the almond-shape fireball produced in the initial stages of a noncentral heavy-ion collision [93] while for $v_{2}$ measured at lower beam energies, the dependences observed are due to interplay of passing time of spectators and time scale of expansion of the system. A preference for an inplane emission versus out-of-plane ("squeeze-out") pattern of particles as a function of beam energy is observed. The experimental data used are from FOPI [94, 95], EOS, E895 [96], E877 [97], CERES [98], NA49 [99], STAR [100], PHOBOS [101], PHENIX [102], ALICE [25], ATLAS [103], and CMS [17-20] experiments. Charged particles are used for LHC, RHIC, CERES, and E877 experiments, pion data is used from NA49 experiment, protons' results are from EOS and E895 experiments, and FOPI results are for all particles with $Z=1$.

3.2.2. Azimuthal Anisotropy Coefficients versus Transverse Momentum. Figure 11(a) shows the comparison of $v_{2}\left(p_{T}\right)$, $v_{3}\left(p_{T}\right)$, and $v_{4}\left(p_{T}\right)$ for $30-40 \%$ collision centrality at RHIC (PHENIX experiment [104]) and LHC (ALICE [105]) at midrapidity in $\mathrm{Au}+\mathrm{Au}$ and $\mathrm{Pb}+\mathrm{Pb}$ collisions, respectively. The bottom panel of this figure shows the ratio of LHC and RHIC results to a polynomial fit to the LHC data. The $v_{n}\left(p_{T}\right)$ measurement techniques are similar at RHIC and LHC energies. One observes that at lower $p_{T}(<2 \mathrm{GeV} / \mathrm{c})$, the $v_{2}\left(p_{T}\right)$ and $v_{3}\left(p_{T}\right)$ are about $10-20 \%$ smaller at RHIC compared to the corresponding LHC results. However, at higher $p_{T}$, the results are quite similar. The $v_{4}\left(p_{T}\right)$ seems higher at RHIC compared to that at LHC.

One of the most striking observations to come out from RHIC is the number of constituent quark $\left(n_{q}\right)$ scaling of $v_{2}\left(p_{T}\right)$ for identified hadrons. The basis of such a scaling is the splitting of $v_{2}\left(p_{T}\right)$ between baryons and mesons at intermediate $p_{T}(2-6 \mathrm{GeV} / \mathrm{c})$. This is shown in the bottom panels of Figure 11(b). Such a splitting between baryon and meson $v_{2}\left(p_{T}\right)$ is also observed at intermediate $p_{T}$ at LHC energies (seen in the top panels of Figure 11(b)). However, the degree to which $n_{q}$ scaling holds could be different at RHIC [106] and LHC [107] energies. The $n_{q}$ scaling is much more closely followed at RHIC compared to LHC. It may be noted that there are several factors which could dilute such scaling, which include energy dependence of radial flow, an admixture of higher Fock states, and consideration of a realistic momentum distribution of quarks inside a hadron $[108,109]$. The observation of the baryon-meson splitting is commonly interpreted as due to substantial amount of collectivity being generated in the deconfined phase. Another important feature is that at both RHIC and LHC energies, a clear hydrodynamic feature of mass dependence of $v_{2}\left(p_{T}\right)$ is observed at low $p_{T}(<2 \mathrm{GeV} / \mathrm{c})$.

Figure 12 shows the charged hadron $v_{2}\left(p_{T}\right)$ for $30-40 \%$ collision centrality in $\mathrm{Au}+\mathrm{Au}$ collisions at $\sqrt{s_{\mathrm{NN}}}=200 \mathrm{GeV}$ and $\mathrm{Pb}+\mathrm{Pb}$ collisions at $\sqrt{s_{\mathrm{NN}}}=2.76 \mathrm{TeV}$ for $|\eta|<1$ [17-20]. This figure demonstrates the kinematic reach for higher energy collisions at LHC relative to RHIC. LHC data allows us to study the $v_{2}\left(p_{T}\right)$ in the $p_{T}$ range never measured 


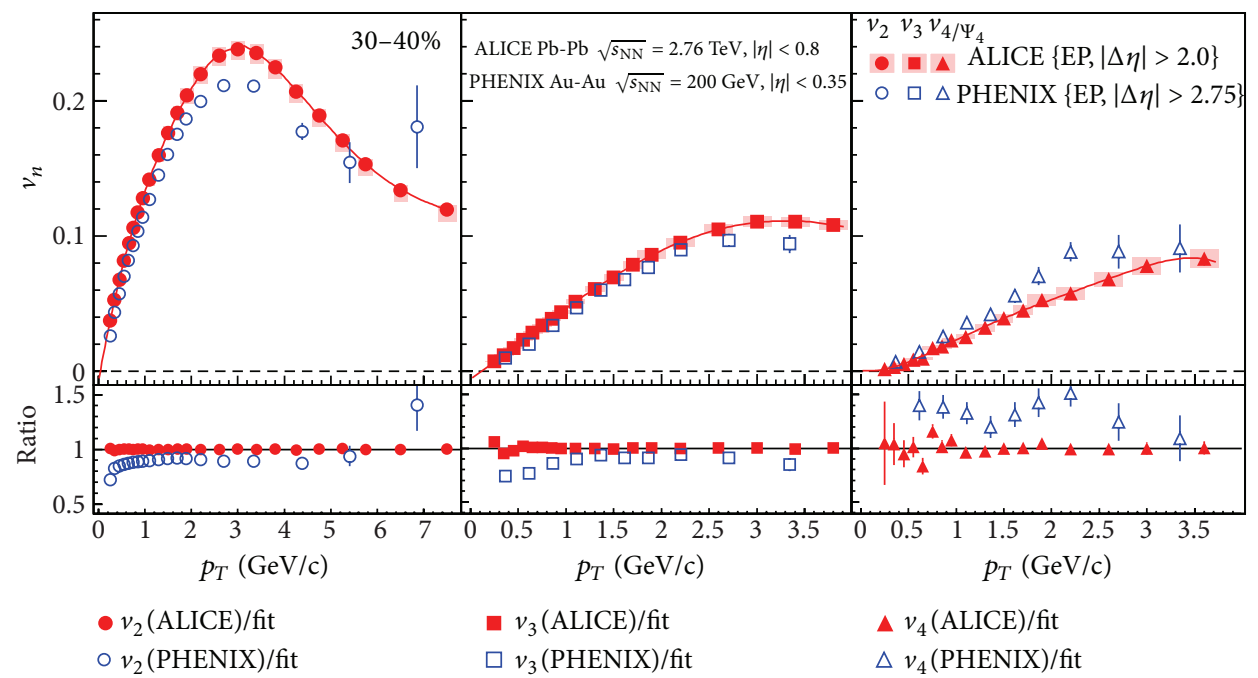

(a)

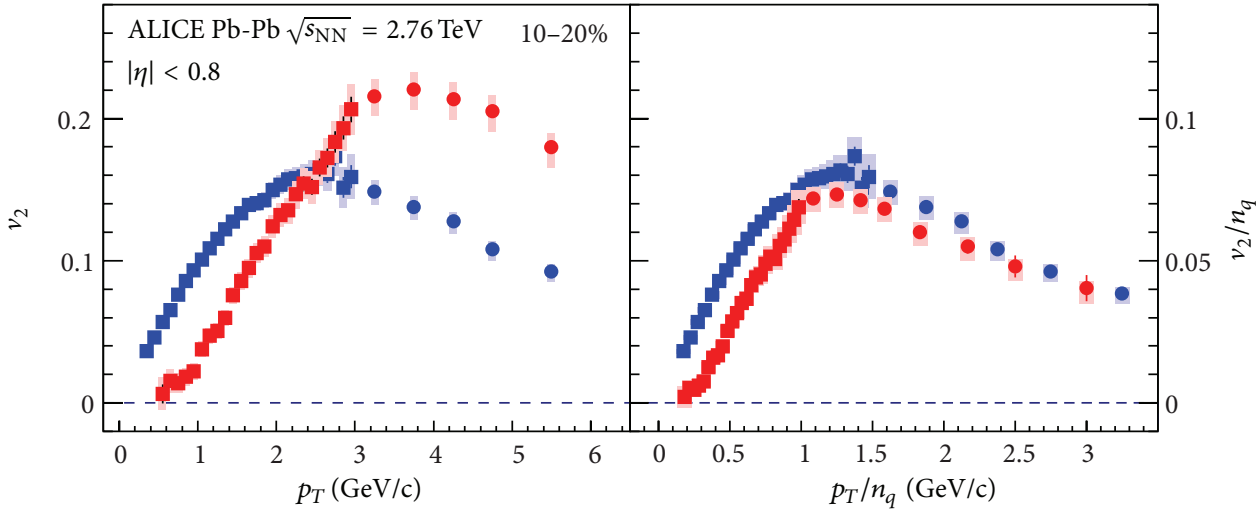

$v_{2}\{\mathrm{SP},|\Delta \eta|>1.0\} \quad v_{2}\{\mathrm{EP},|\Delta \eta|>2.0\}$

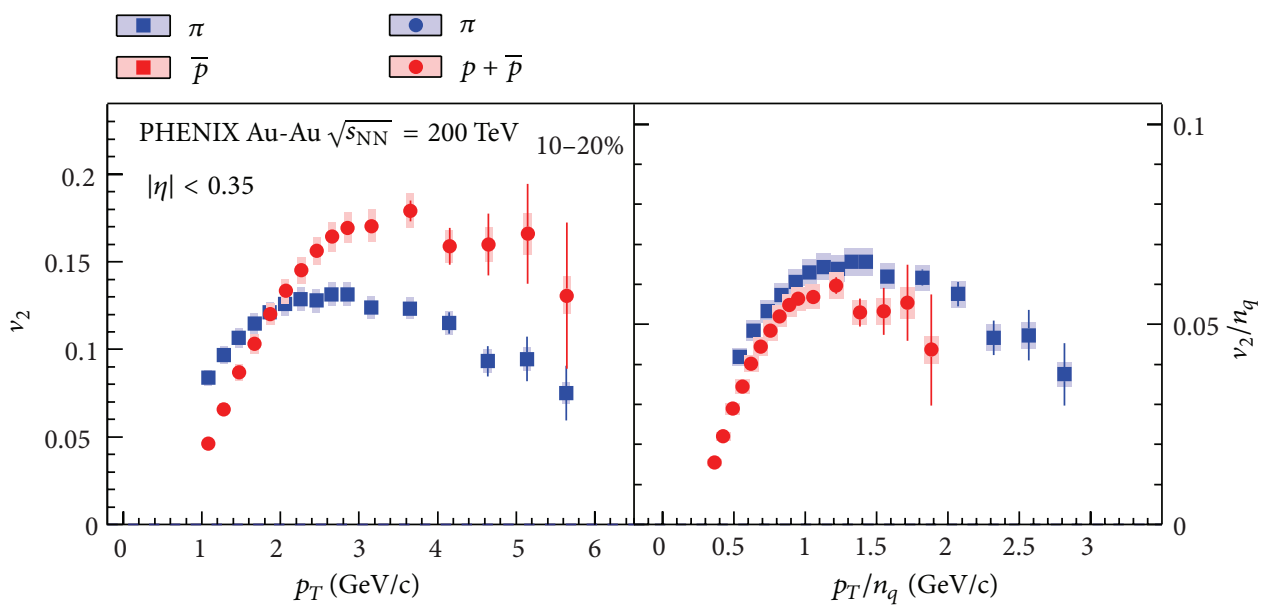

$v_{2}\{\mathrm{EP},|\Delta \eta|>2.75\}$

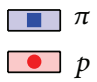

(b)

FIGURE 11: (Color online) (a) Comparison of $v_{n}\left(p_{T}\right)$ at midrapidity for 30-40\% collision centrality at RHIC $\left(\mathrm{Au}+\mathrm{Au}\right.$ collisions at $\sqrt{s_{\mathrm{NN}}}=$ $200 \mathrm{GeV}$ from PHENIX experiment [104]) and at LHC (Pb $+\mathrm{Pb}$ collisions at $\sqrt{\boldsymbol{s}_{\mathrm{NN}}}=2.76 \mathrm{TeV}$ from ALICE experiment [105]). (b) show the ratio of $v_{n}$ at LHC and RHIC. (b) $v_{2}$ versus $p_{T}$ and $v_{2} / n_{q}$ versus $p_{T} / n_{q}$ for pions and protons at midrapidity for $10-20 \%$ collision centrality from $\mathrm{Au}+\mathrm{Au}$ collisions at $\sqrt{s_{\mathrm{NN}}}=200 \mathrm{GeV}$ (PHENIX experiment [106]) and $\mathrm{Pb}+\mathrm{Pb}$ collisions at $\sqrt{s_{\mathrm{NN}}}=2.76 \mathrm{TeV}$ (ALICE experiment $[107])$. 


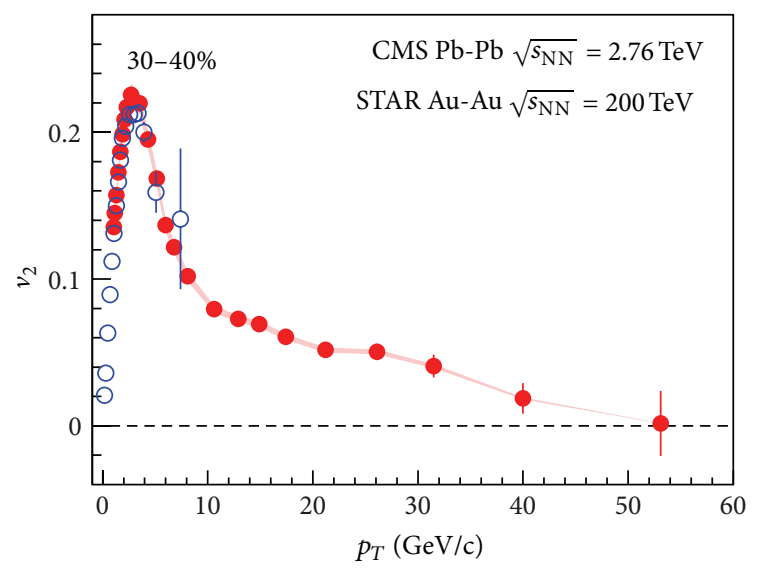

CMS $\{\mathrm{EP}\},|\eta|<1.0$

○ $\operatorname{STAR}\{\mathrm{EP}\},|\eta|<1.0$

FIgURE 12: (Color online) Comparison of $v_{2}\left(p_{T}\right)$ at midrapidity for $30-40 \%$ collision centrality at RHIC $\left(\mathrm{Au}+\mathrm{Au}\right.$ collisions at $\sqrt{s_{\mathrm{NN}}}=$ $200 \mathrm{GeV}$ from STAR experiment) and at LHC ( $\mathrm{Pb}+\mathrm{Pb}$ collisions at $\sqrt{s_{\mathrm{NN}}}=2.76 \mathrm{TeV}$ from CMS experiment [17-20]). The shaded band about CMS data point are systematic errors and vertical lines represent statistical errors.

before in heavy-ion collisions. The $v_{2}\left(p_{T}\right) \sim 0$ for $p_{T}>$ $40 \mathrm{GeV} / \mathrm{c}$ might suggest that those particles must have been emitted very early in the interactions when the collective effects had not set in. These high transverse momentum data are useful to understand the effects of the initial geometry or path-length dependence of various properties associated with parton modification inside the hot QCD medium. In addition, it also provides significantly improved precision measurement of $v_{2}$ for $12<p_{T}<20 \mathrm{GeV} / \mathrm{c}$.

3.2.3. Flow Fluctuations. Fluctuations in azimuthal anisotropy coefficient $v_{2}$ have gained quite an attention in recent times. In particular, the measurement of event-by-event $v_{2}$ fluctuations can pose new constraints on the models of the initial state of the collision and their subsequent hydrodynamic evolution. In extracting event-by-event $v_{2}$ fluctuations, one needs to separate nonflow effects, and so far, there is no direct method to decouple $v_{2}$ fluctuations and nonflow effects in a model independent from the experimental measurements. However, several techniques exist where the nonflow effects can be minimized; for example, flow and non-flow contributions can be possibly separated to a great extent with a detailed study of two particle correlation function in $\Delta \phi$ and its dependence on $\eta$ and $\Delta \eta$. Here, we discuss another technique to extract and compare the $v_{2}$ fluctuations at RHIC and LHC. We assume that the difference between $v_{2}\{2\}$ (two-particle cumulant) and $v_{2}\{4\}$ (four-particle cumulant) is dominated by $v_{2}$ fluctuations, and nonflow effect is negligible for $v_{2}\{4\}$. Then, the ratio $R_{v(2-4)}=$ $\sqrt{\left(v_{2}\{2\}^{2}-v_{2}\{4\}^{2}\right) /\left(v_{2}\{2\}^{2}+v_{2}\{4\}^{2}\right)}$ can be considered as an estimate for $v_{2}$ fluctuations in the data. Figure 13 shows the
$R_{v(2-4)}$ as a function of collision centrality and $\left\langle d N_{\mathrm{ch}} / d \eta\right\rangle$ for RHIC [110] and LHC [107] energies. The centrality dependence of $R_{v(2-4)}$ at RHIC or LHC as seen in Figure 13 could be an interplay of residual nonflow effects which increases for central collisions and multiplicity fluctuations which dominate smaller systems. It is striking to see that $R_{v(2-4)}$ when presented as a function of \% cross section is similar at RHIC and LHC, suggesting it reflects features associated with initial state of the collisions, for example, the event-by-event fluctuations in the eccentricity of the system. But when presented as a function of $d N_{\mathrm{ch}} / d \eta$, it tends to suggest a different behavior for most central collisions at RHIC.

Recently, a great interest has been generated on extracting initial condition and flow fluctuation information from the measurement of the probability distribution of $v_{n}$ at LHC. The probability density of $v_{n}$ can be expressed as a Gaussian function in transverse plane [111] as $p\left(v_{n}\right)=\left(1 / 2 \pi \delta_{v_{n}}^{2}\right) e^{-\left(v_{n}-v_{n}^{\mathrm{RP}}\right)^{2} /\left(2 \delta_{v_{n}}^{2}\right)}$ or as one dimensional Bessel-Gaussian function [112, 113] as $p\left(v_{n}\right)=\left(v_{n} /\right.$ $\left.\delta_{v_{n}}^{2}\right) e^{-\left(\left(\left(v_{n}\right)^{2}+\left(v_{n}^{\mathrm{RP}}\right)^{2}\right) / 2 \delta_{v_{n}}^{2}\right)} I_{0}\left(v_{n}^{\mathrm{RP}} v_{n} / \delta_{v_{n}}^{2}\right)$, where $I_{0}$ is the modified Bessel function of the first kind and $\delta_{v_{n}}$ is the fluctuation in $v_{n}$, with $\delta_{v_{n}} \approx \sigma_{v_{n}}$ for $\delta_{v_{n}} \ll v_{n}^{\mathrm{RP}}\left(v_{n}\right.$ measured with respect to reaction plane).

Figure 14 shows the $v_{2}^{\mathrm{RP}}$ and $\delta_{v_{2}}$ values extracted from the $v_{2}$ distributions as a function of $\left\langle N_{\text {part }}\right\rangle$ by fitting to the above probability functions [114]. They are compared with values of $\left\langle v_{2}\right\rangle$ and $\sigma_{v_{2}}$ obtained directly from the $v_{2}$ distributions. The $v_{2}^{\mathrm{RP}}$ value is always smaller than the value for $\left\langle v_{2}\right\rangle$, and it decreases to zero in the $0-2 \%$ centrality interval. The value of $\delta_{v_{2}}$ is close to $\sigma_{v_{2}}$, except in the most central collisions. This leads to a value of $\delta_{v_{2}} / v_{2}^{\mathrm{RP}}$ larger than $\sigma_{v_{2}} /\left\langle v_{2}\right\rangle$ over the full centrality range as shown in Figure 14(c). The value of $\delta_{v_{2}} / v_{2}^{\mathrm{RP}}$ decreases with $\left\langle N_{\text {part }}\right\rangle$ and reaches a minimum at $\left\langle N_{\text {part }}\right\rangle \approx$ 200 but then increases for more central collisions. Thus, the event-by-event $v_{2}$ distribution brings additional insight for the understanding of $v_{2}$ fluctuations.

3.3. Nuclear Modification Factor. Figure 15 shows the $R_{\mathrm{AA}}$ of various particles produced in heavy-ion collisions at RHIC and LHC. In Figure 15(a), we observe that the shape of the $R_{\mathrm{AA}}$ versus $p_{T}$ of charged hadrons at RHIC and LHC $[26,27]$ is very similar for the common $p_{T}$ range of measurements. The values $R_{\mathrm{AA}}$ at RHIC are higher compared to those at LHC energies up to $p_{T}<8 \mathrm{GeV} / \mathrm{c}$. The higher kinematic reach of LHC in $p_{T}$ allows us to see the full $p_{T}$ evolution of $R_{\mathrm{AA}}$ in high energy heavy-ion collisions. All these measurements suggest that the energy loss of partons in the medium formed in heavy-ion collisions at LHC energies is perhaps larger compared to that at RHIC. In Figure 15(b), we observe that the nuclear modification factors for $d+$ Au collisions at $\sqrt{s_{\mathrm{NN}}}=200 \mathrm{GeV}$ [115] and $p+\mathrm{Pb}$ collisions at $\sqrt{s_{\mathrm{NN}}}=$ $5.02 \mathrm{TeV}[116]$ are greater than unity for the $p_{T}>2 \mathrm{GeV} / \mathrm{c}$. The values for RHIC are slightly larger compared to those for LHC. A value greater than unity for the nuclear modification factor in $p(d)+$ A collisions is generally interpreted as due to 


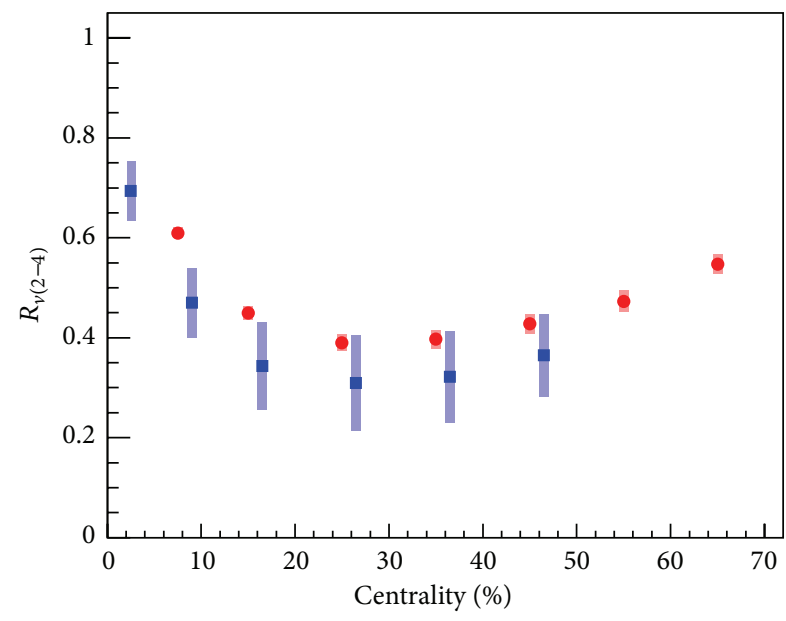

- $\mathrm{Au}+\mathrm{Au} \sqrt{s_{\mathrm{NN}}}=0.2 \mathrm{TeV}(|\eta|<1)$

1. $\mathrm{Pb}+\mathrm{Pb} \sqrt{s_{\mathrm{NN}}}=2.76 \mathrm{TeV}(|\eta|<2)$

(a)

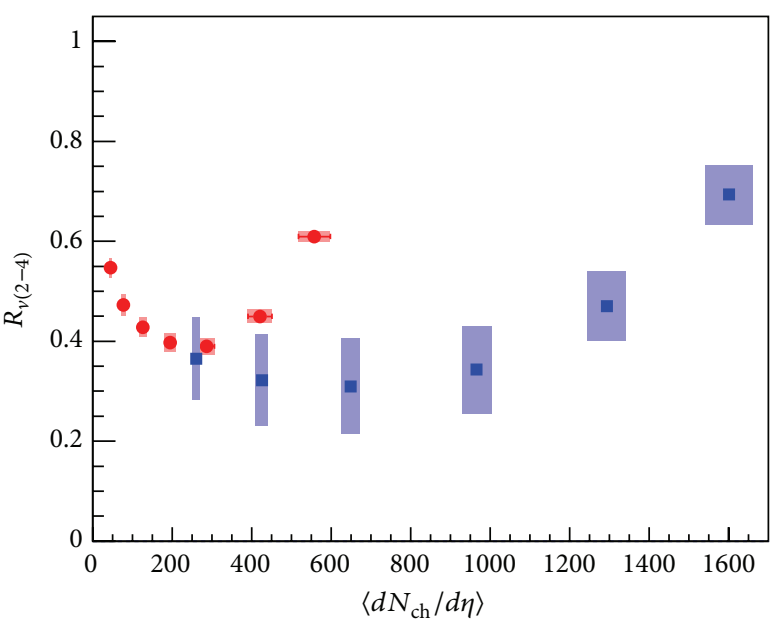

$\mathrm{Au}+\mathrm{Au} \sqrt{s_{\mathrm{NN}}}=0.2 \mathrm{TeV}(|\eta|<1)$

$\mathrm{Pb}+\mathrm{Pb} \sqrt{s_{\mathrm{NN}}}=2.76 \mathrm{TeV}(|\eta|<2)$

(b)

FIGURE 13: (Color online) The ratio $R_{v(2-4)}=\sqrt{\left(v_{2}\{2\}^{2}-v_{2}\{4\}^{2}\right) /\left(v_{2}\{2\}^{2}+v_{2}\{4\}^{2}\right)}$, an estimate of $v_{2}$ fluctuations plotted as a function of collision centrality (a) and $\left\langle d N_{\mathrm{ch}} / d \eta\right\rangle$ (b) for RHIC (STAR experiment: Au + Au collisions at $\sqrt{s_{\mathrm{NN}}}=200 \mathrm{GeV}$ [110]) and LHC (ALICE: $\mathrm{Pb}+\mathrm{Pb}$ collisions at $\sqrt{s_{\mathrm{NN}}}=2.76 \mathrm{TeV}$ [107]) at midrapidity. The bands reflect the systematic errors.

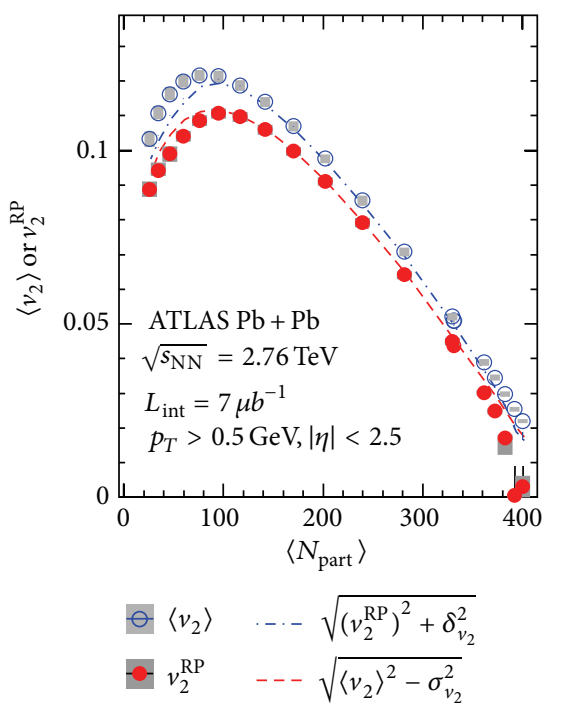

(a)

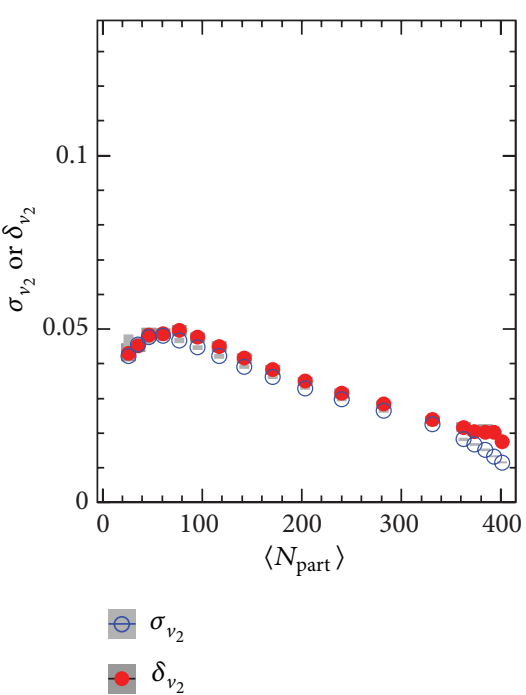

(b)

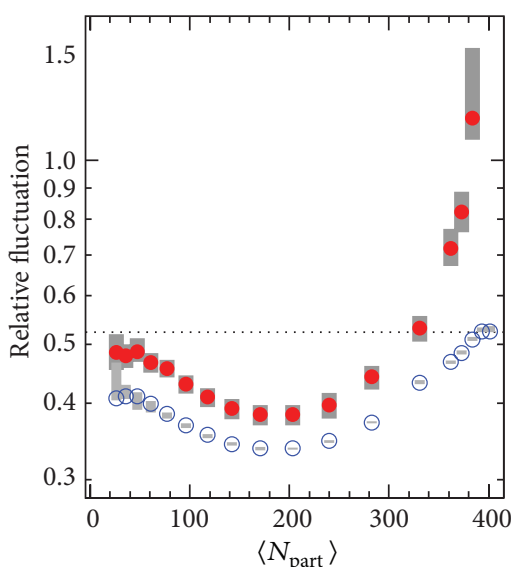

$\ominus \sigma_{v_{2}} /\left\langle v_{2}\right\rangle$

$\delta_{v_{2}} / v_{2}^{\mathrm{RP}}$

(c)

Figure 14: (Color online) The dependence of $v_{2}^{\mathrm{RP}}$ and $\left\langle v_{2}\right\rangle(\mathrm{a}), \delta_{v_{2}}$ and $\sigma_{v_{2}}$ (b), and $\delta_{v_{2}} / v_{2}^{\mathrm{RP}}$ and $\sigma_{v_{2}} /\left\langle v_{2}\right\rangle$ (c) on $\left\langle N_{\text {part }}\right\rangle$ [114]. The shaded boxes indicate the systematic uncertainties.

Cronin effect $[117,118]$. However, several other physics effects could influence the magnitude of the nuclear modification factor in $p(d)+$ A collisions such as nuclear shadowing and gluon saturation effects. But the results that the nuclear modification factors in $p(d)+\mathrm{A}$ collisions are not below unity strengthen the argument (from experimental point of view) that a hot and dense medium of color charges is formed in A + A collisions at RHIC and LHC. In Figure 15(c), we show the $R_{\mathrm{AA}}$ of particles that do not participate in strong interactions, and some of them are most likely formed in the 


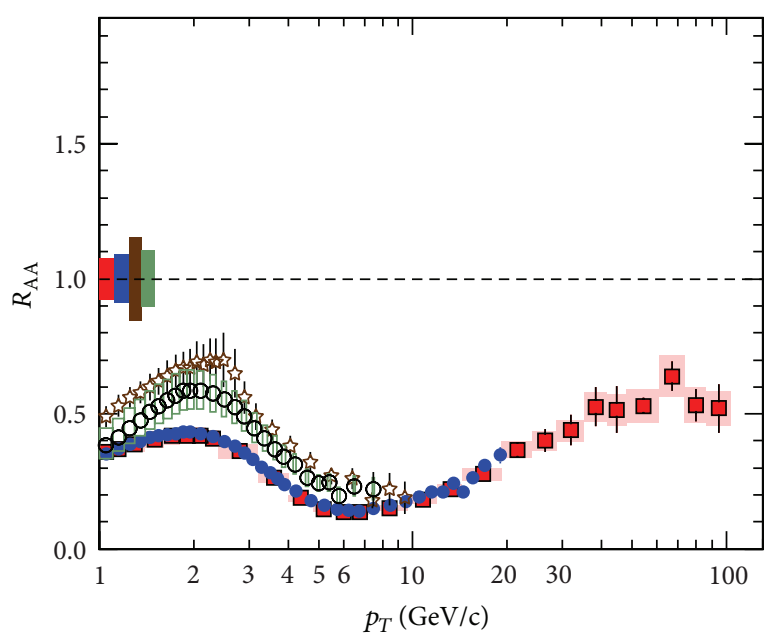

- $h^{ \pm} \mathrm{CMSPbPb}(0-5 \%) \sqrt{s_{\mathrm{NN}}}=2.76 \mathrm{TeV}$

- $h^{ \pm}$ALICE PbPb $(0-5 \%) \sqrt{s_{\mathrm{NN}}}=2.76 \mathrm{TeV}$

的 $h^{ \pm}$STAR AuAu $(0-5 \%) \sqrt{s_{\mathrm{NN}}}=0.2 \mathrm{TeV}$

O $\pi^{0}$ PHENIX AuAu $(0-10 \%) \sqrt{s_{\mathrm{NN}}}=0.2 \mathrm{TeV}$

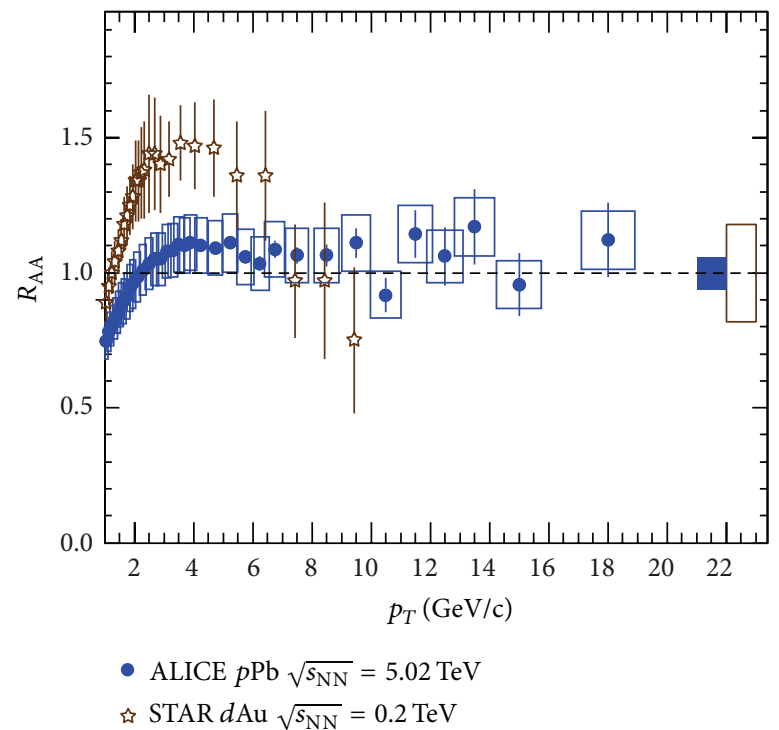

(b)

(a)

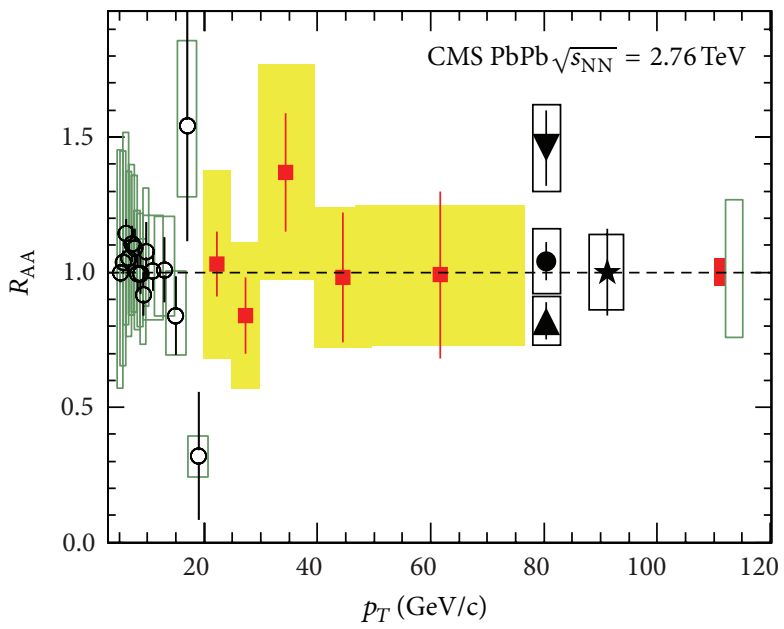

Isolated photon

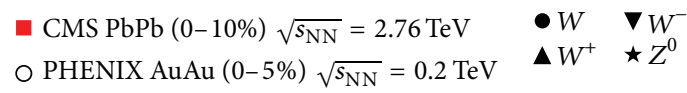

(c)

Figure 15: (Color online) (a) Nuclear modification factor $R_{\mathrm{AA}}$ of charged hadrons measured by ALICE [26] and CMS [27] experiments at midrapidity for $0-5 \%$ most central $\mathrm{Pb}+\mathrm{Pb}$ collisions at $\sqrt{s_{\mathrm{NN}}}=2.76 \mathrm{TeV}$. For comparison, shown are the $R_{\mathrm{AA}}$ of charged hadrons at midrapidity for $0-5 \%$ most central collisions measured by STAR [115] and $R_{\mathrm{AA}}$ of $\pi^{0}$ at midrapidity for $0-10 \%$ most central collisions measured by PHENIX [173] for $\mathrm{Au}+\mathrm{Au}$ collisions at $\sqrt{s_{\mathrm{NN}}}=200 \mathrm{GeV}$. (b) Comparison of nuclear modification factor for charged hadrons versus $p_{T}$ at midrapidity for minimum bias collisions in $d+$ Au collisions at $\sqrt{s_{\mathrm{NN}}}=200 \mathrm{GeV}$ [115] and $p+\mathrm{Pb}$ collisions at $\sqrt{s_{\mathrm{NN}}}=5.02 \mathrm{TeV}$ [116]. (c) The nuclear modification factor versus $p_{T}$ for isolated photons in central nucleus-nucleus collisions at $\sqrt{s_{\mathrm{NN}}}=200 \mathrm{GeV}[119]$ and $2.76 \mathrm{TeV}$ [120]. Also shown are the $p_{T}$ integrated $R_{\mathrm{AA}}$ of $W^{ \pm}$[121] and $Z$ bosons [122] at corresponding $m_{T}$ at LHC energies. Open and shaded boxes represents the systematic uncertainties in the experimental measurements and normalization uncertainties, respectively.

very early stages of the collisions. These particles (photon $[119,120], W^{ \pm}[121]$, and $Z[122]$ bosons) have an $R_{\mathrm{AA}} \sim 1$, indicating that the $R_{\mathrm{AA}}<1$, observed for charged hadrons in $\mathrm{A}+\mathrm{A}$ collisions, is due to the strong interactions in a dense medium consisting of color charges.

\section{Comparison to Model Calculations}

In this section, we compare some of the experimental observables discussed above with corresponding model calculations. This helps us to interpret the data at both RHIC and 

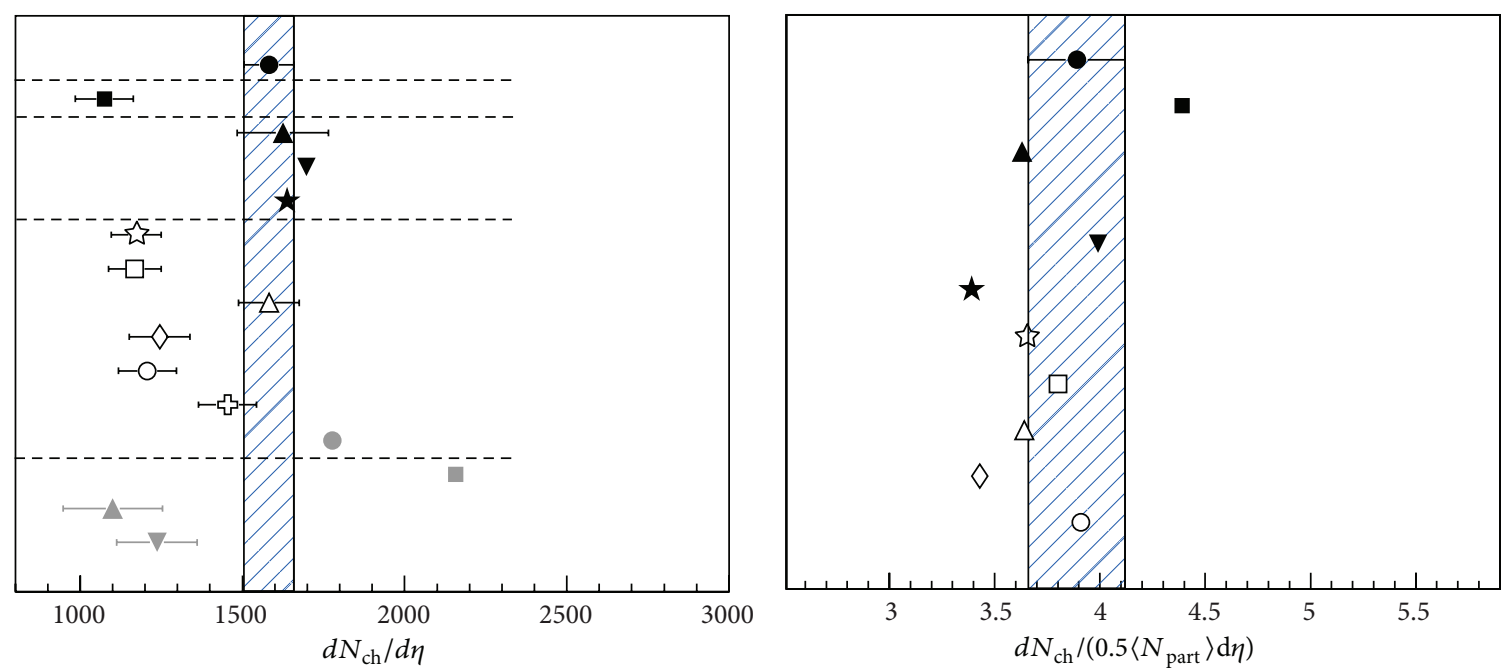

- ALICE
$\square$ Busza
$\Delta$ HIJING 2.0
$\nabla$ DPMJET III
$\star$ UrQMD
$\lesssim$ Albacete
$\square$ Levin
$\Delta$ Kharzeev1

\section{$\diamond$ Kharzeev2 \\ O Kharzeev3 \\ や Armesto \\ - Eskola \\ Bozek \\ $\triangle$ Sarkisyan \\ $\nabla$ Humanic}

(a)

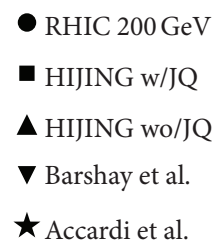

ఓjeon and Kapusta

$\square$ Eskola et al.

$\triangle$ Dias de Deus

$\diamond$ D. E. Kahana and S. H. kahana

O Armesto et al.

(b)

FIGURE 16: (Color online) Comparison of $d N_{\mathrm{ch}} / d \eta$ measurement at midrapidity for central heavy-ion collisions at RHIC and LHC with model predictions.

LHC energies. We restrict our discussion on the comparison of the models with the experimental data for charged particle production, ratio of kaon to pion yields as a function of beam energy, $p_{T}$ dependence of $v_{2}$, and $R_{\mathrm{AA}}$ for charged particles and pions. For the charged particle production, we compare the experimental data with models inspired by the perturbative QCD-based calculations (HIJING, DPMJET) with macroscopic models (statistical and hydrodynamical), microscopic models (string, transport, cascade, etc.), and calculations which are derived by the different parametrizations of the nucleon-nucleon and nucleus-nucleus lower energy data. The ratio of kaon to pion yields for different beam energies is compared with the statistical and thermal models. The transverse momentum dependence of $v_{2}$ is compared with models incorporating the calculations based on hydrodynamic and transport approaches. Finally, the $R_{\mathrm{AA}}$ results are compared with the perturbative QCD-based calculations with different mechanism for the parton energy loss in the presence of colored medium.

4.1. Charged Particle Multiplicity Density and Particle Ratio. Figure 16 compares the measured charged particle pseudorapidity density at RHIC $(0.2 \mathrm{TeV})$ and LHC (2.76 TeV) energies to various model calculations.

Empirical extrapolation from lower energy data (named "Busza" in the figure) [123] significantly under-predicts the measurement at LHC energies. A simple power-law growth of charged-particle multiplicities near midrapidity in central
$\mathrm{Au}+\mathrm{Au}$ collisions seems to be followed up to RHIC energies (named as "Barshay and Kreyerhoff" in the figure) [124]. Perturbative QCD-inspired Monte Carlo event generators, the HIJING model without jet quenching [125], the Dual Parton Model [126] (named "DPMJET III" in the figure), and the Ultrarelativistic Quantum Molecular Dynamics model [127] (named "UrQMD" in the figure) are consistent with the measurement. The HIJING model results without jet quenching were also consistent with the RHIC measurements. The semimicroscopic models like LEXUS are successful in explaining the observed multiplicity at RHIC (named as "Jeon and Kapusta" in the figure) [128]. Models based on initialstate gluon density saturation have a range of predictions depending on the specifics of the implementation [129-133]. The best agreement with LHC data happens for model as described in (named as "Kharzeev et al." and "Armesto et al." in the figure) $[131,133]$. Conclusions for RHIC energy from these models are similar. The prediction of a hybrid model based on hydrodynamics and saturation of final-state phase space of scattered partons (named as "Eskola et al." in the figure) [134] is slightly on a higher side compared to the measurement at LHC. But such a model seems to do a reasonable job for RHIC energies [135]. Another hydrodynamic model in which multiplicity is scaled from $p+p$ collisions overpredicts the measurement (named as "Bozek et al." in the figure) [136]. Models incorporating constituent quark scaling and Landau hydrodynamics (named as "Sarkisyan and Sakharov" in the figure) $[137,138]$ and based on modified PYTHIA 


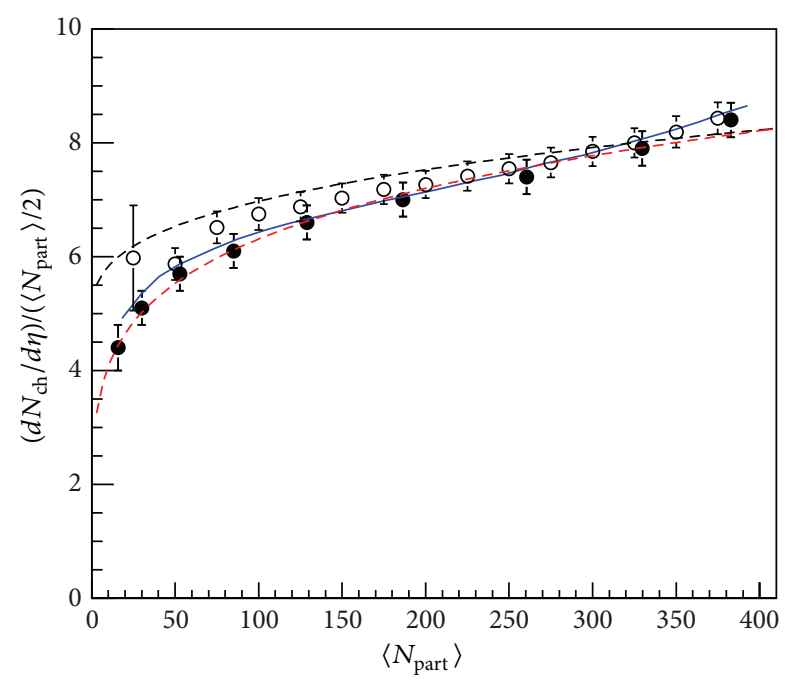

- ALICE Pb + Pb 2.76 TeV

- $\mathrm{RHIC} \mathrm{Au}+\mathrm{Au} 0.20 \mathrm{TeV}(\times 2.15)$

- - $\left[0.0147 \ln (s * s)^{2}+0.6\right]\left(1+0.095 N_{\text {part }}^{1 / 3}\right)(\times 2.15)$

$---2.63 N_{\text {part }}^{0.19}$

_ Albacete et al.

Figure 17: (Color online) Centrality dependence of $\left(d N_{\mathrm{ch}} / d \eta\right) /\left(\left\langle N_{\text {part }}\right\rangle / 2\right)$ for $\mathrm{Pb}+\mathrm{Pb}$ collisions at $\sqrt{s_{\mathrm{NN}}}=2.76 \mathrm{TeV}$ [14] and $\mathrm{Au}+\mathrm{Au}$ collisions at $\sqrt{s_{\mathrm{NN}}}=200 \mathrm{GeV}$. The RHIC results are scaled up by a factor of 2.15 . Also shown are comparisons to theoretical model calculations [144] and some parametrization based on detail shape of $d N_{\mathrm{ch}} / d \eta$ distributions at RHIC [47] and $\left\langle N_{\text {part }}\right\rangle$.

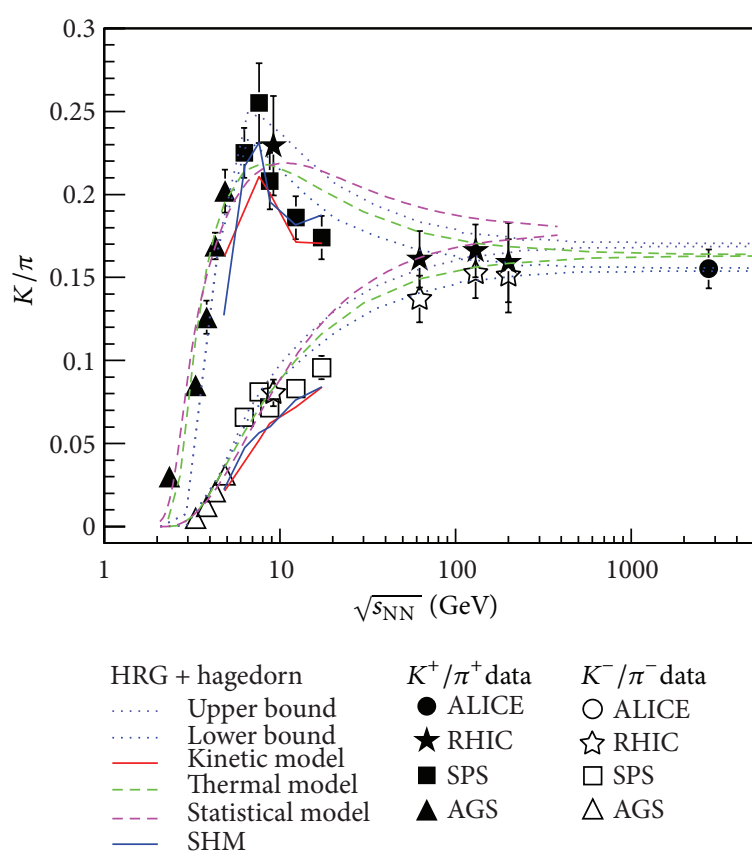

FIGURE 18: (Color online) Energy dependence of $K^{ \pm} / \pi^{ \pm}$ratio for central collisions at midrapidity. Errors are statistical and systematic added in quadrature. Results are also compared with various theoretical model predictions [79, 147-150]. and hadronic re-scattering (named as "Humanic" in the figure) [139] underpredict the measurement at LHC energy. At RHIC energies, models considering minijet production in ultrarelativistic heavy-ion collisions by taking semihard parton rescatterings explicitly into account underpredict the multiplicities (named as "Accardi" in the figure) [140]. It is also seen at RHIC energies that models based on string fusion [141] and dual string model [142] seem to work well, whereas those based on heavy-ion cascade LUCIFER model [143] underpredict the data.

Figure 17 shows the $\left(d N_{\mathrm{ch}} / d \eta\right) /\left(\left\langle N_{\text {part }}\right\rangle / 2\right)$ versus $\left\langle N_{\text {part }}\right\rangle$ for $\mathrm{Pb}+\mathrm{Pb}$ collisions at $\sqrt{s_{\mathrm{NN}}}=2.76 \mathrm{TeV}$ [14]. Also shown are the corresponding RHIC results scaled up by a factor 2.15. Remarkable similarity is observed in the shape of the distributions at RHIC and LHC energies. Particle production based on saturation model explains the trends nicely (named as "ALbacete and Dumitru" in the figure) [144] (published after the most central $d N_{\text {ch }} / d \eta$ value [25] was known). simple fit to the data using a power law form for the $\left\langle N_{\text {part }}\right\rangle$ also explains the measurements. In addition, a functional form inspired by the detailed shape of pseudorapidity distribution of charged particle multiplicity distributions at RHIC [47] explains the centrality trends nicely.

Strangeness production in heavy-ion collisions is a classic signature for formation of QGP [145]. The particle yield ratio $K / \pi$ could reflect the strangeness enhancement in heavy-ion collisions with respect to the elementary collisions. Figure 18 shows the energy dependence of $K^{ \pm} / \pi^{ \pm}$ratio for central collisions at midrapidity. It will be interesting to see which model explains such an impressive collection of systematic data on $K / \pi$ ratio. Figure 18 also shows the energy dependence of $K / \pi$ ratio from various theoretical model calculations. The energy dependence of $K^{+} / \pi^{+}$ratio has been interpreted using the Statistical Model of Early Stage (SMES) [146]. The model predicts first-order phase transition and the existence of mixed phase around beam energy of 7-8 GeV. The SHM or statistical hadronization model [147] assumes that the strong interactions saturate the particle production matrix elements. This means that the yield of particles is controlled predominantly by the magnitude of the accessible phase space. The system is in chemical nonequilibrium for $\sqrt{s_{\mathrm{NN}}}<7.6 \mathrm{GeV}$, while for higher energies, the oversaturation of chemical occupancies is observed. The statistical model [148] assumes that the ratio of entropy to $T^{3}$ as a function of collision energy increases for mesons and decreases for baryons. Thus, a rapid change is expected at the crossing of the two curves, as the hadronic gas undergoes a transition from a baryon-dominated to a meson-dominated gas. The transition point is characterized by $T=140 \mathrm{MeV}, \mu_{B}=$ $410 \mathrm{MeV}$, and $\sqrt{s_{\mathrm{NN}}}=8.2 \mathrm{GeV}$. In the thermal model [79], the energy dependence of $K^{ \pm} / \pi^{ \pm}$is studied by including $\sigma$ meson, which is neglected in most of the models, and many higher mass resonances $\left(m>2 \mathrm{GeV} / \mathrm{c}^{2}\right)$ into the resonance spectrum employed in the statistical model calculations. The hadronic nonequilibrium kinetic model [149] assumes that the surplus of strange particles is produced in secondary reactions of hadrons generated in nuclear collisions. Then, the two important aspects are the available energy density and the 
lifetime of the fireball. It is suggested that these two aspects combine in such a way so as to show a sharp peak for the strangeness-to-entropy or $K / \pi$ ratio as a function of beam energy. In the hadron resonance gas and hagedorn (HRG + Hagedorn) model [150], all hadrons as given in PDG with masses up to $2 \mathrm{GeV} / \mathrm{c}^{2}$ are included. The unknown hadron resonances in this model are included through Hagedorn's formula for the density of states. The model assumes that the strangeness in the baryon sector decays to strange baryons and does not contribute to the kaon production. The energy dependence of $K^{ \pm} / \pi^{ \pm}$ratio seems to be best explained using HRG + Hagedorn model.

This systematic measurement of $K / \pi$ ratio reveals two interesting pieces of information. (a) The $K^{+} / \pi^{+}$ratio shows a peak around $\sqrt{s_{\mathrm{NN}}}=8 \mathrm{GeV}$, while the $K^{-} / \pi^{-}$ratio increases monotonically; the peak indicates the role of the maximum baryon density at freeze-out around this collision energy. (b) For $\sqrt{s_{\mathrm{NN}}}>100 \mathrm{GeV}$, pair production becomes the dominant mechanism for $K^{ \pm}$production, so both the ratios $K^{+} / \pi^{+}$and $K^{-} / \pi^{-}$approach the value of 0.16 . Taking into account the different masses between pions and kaons, this asymptotic value corresponds to a temperature of the order of $160 \mathrm{MeV}$.

4.2. Azimuthal Anisotropy. The azimuthal anisotropy parameter $v_{2}$, measured at RHIC and LHC, provides a unique opportunity to study the transport properties of the fundamental constituents of any visible matter, a system of quarks and gluons. Furthermore, it provides an opportunity to understand whether the underlying dynamics of the evolution of the system formed in the collisions are governed by macroscopic hydrodynamics [151-153] or by microscopic transport approach [154]. Figure 19 shows the $v_{2}$ versus $p_{T}$ for $30-40 \%$ collision centrality $\mathrm{Au}+\mathrm{Au}$ and $\mathrm{Pb}+\mathrm{Pb}$ collisions at midrapidity for $\sqrt{s_{\mathrm{NN}}}=200 \mathrm{GeV}$ and $2.76 \mathrm{TeV}$, respectively. The measurements are compared to a set of model calculations based on hydrodynamic approach (including THERMINATOR $[155,156])$ and another set of calculations based on transport approach. It is observed that hydrodynamic-based models explain the $v_{2}$ measurements both at RHIC and LHC energies. Transport-based models including partonic interactions (like AMPT [154]) also explain the $v_{2}$ measurements. However, those transport models which do not incorporate partonic interactions like UrQMD $[157,158]$ fail to explain the data. The model comparison also reveals that the data favors a high degree of fluidity reflected by a small value of shear viscosity to entropy density ratio $(\eta / s)<0.2$. A more detailed comparison of the model calculations with various order azimuthal anisotropy parameters $v_{n}$ would in the near future give us a more quantitative picture of the temperature (or energy) dependence of transport coefficients of the system formed in the heavy-ion collisions.

4.3. Nuclear Modification Factor. The nuclear modification factor $\left(R_{\mathrm{AA}}\right)$ is an observable used to study the structure of strongly interacting dense matter formed in heavy-ion collisions. Here, we discuss the observation of $R_{\mathrm{AA}}<1$ at high $p_{T}$ seen at RHIC and LHC by comparing two models within perturbative QCD- (pQCD-) based formalisms. In this picture, the high $p_{T}$ hadrons are expected to originate from the fragmentation of hard partons (hard scattering scales larger than QCD scales of $200 \mathrm{MeV}$ ). The hard partons lose energy through interactions with the hot and dense mediums, which get reflected in the observed values of $R_{\mathrm{AA}}$. The processes by which they could lose energy includes radiative energy loss and elastic energy loss. For a more elaborate discussion on these models, we refer the reader to the review article [159].

In Figure 20, we show a comparison between experimentally measured $R_{\mathrm{AA}}$ versus $p_{T}$ at LHC and RHIC energies and corresponding pQCD-based model calculations. All theoretical formalisms require a microscopic model of the medium to set the input parameters for the energy loss calculation. These parameters, for example, are denoted as $\langle\widehat{q}\rangle$, the transport coefficient of the medium or the gluon number density $d N^{g} / d y$ per unit rapidity. The parameter $P_{\text {esc }}$, on the other hand, reflects the strength of elastic energy loss put in the model calculations. Without going into deeper theoretical discussions of each model, we refer the readers to the following related publications: PQM [160], GLV [161], ASW [162], YaJEM [163], WHDG [164], and ZOWW [165]. However, for completeness and to elucidate the approach taken in the model calculations, we briefly mention two formalisms as examples: the GLV approach named after their authors Gyulassy, Levai, and Vitev and ASW approach named after the corresponding authors Armesto, Salgado and Wiedemann, where the medium is defined as separated heavy static scattering centers with color screened potentials, where as in some other formalism, a more precise definition of the medium is considered as being composed of quark gluon quasiparticles with dispersion relations and interactions given by the hard thermal loop effective theory.

We observe that most models predict the $p_{T}$ dependence of $R_{\mathrm{AA}}$ well for collisions both at RHIC and LHC energies. The models specially capture the generally rising behavior of $R_{\mathrm{AA}}$ that is observed in the data at high $p_{T}$ for the LHC energies. The magnitude of the predicted slope of $R_{\mathrm{AA}}$ versus $p_{T}$ varies between models, depending on the assumptions for the jet-quenching mechanism. The models shown do not need larger values of medium density in the calculation to explain the $R_{\mathrm{AA}}$ for $3<p_{\mathrm{T}}<20 \mathrm{GeV} / \mathrm{c}$ at RHIC and LHC for the common kinematic range. They however, require a high medium density at LHC energy to explain the values of $R_{\mathrm{AA}}$ for $p_{T}>20 \mathrm{GeV} / \mathrm{c}$.

\section{Summary}

In summary, the results on multiplicity density in pseudorapidity, HBT, azimuthal anisotropy, and nuclear modification factor from LHC experiments indicate that the fireball produced in these nuclear collisions is hotter, lives longer, and expands to a larger size at freeze-out compared to lower energies. These results also confirm the formation of a deconfined state of quarks and gluons at RHIC energies. The measurements at LHC provide a unique kinematic access to study in detail the properties (such as transport coefficients) of this system of quarks and gluons. 


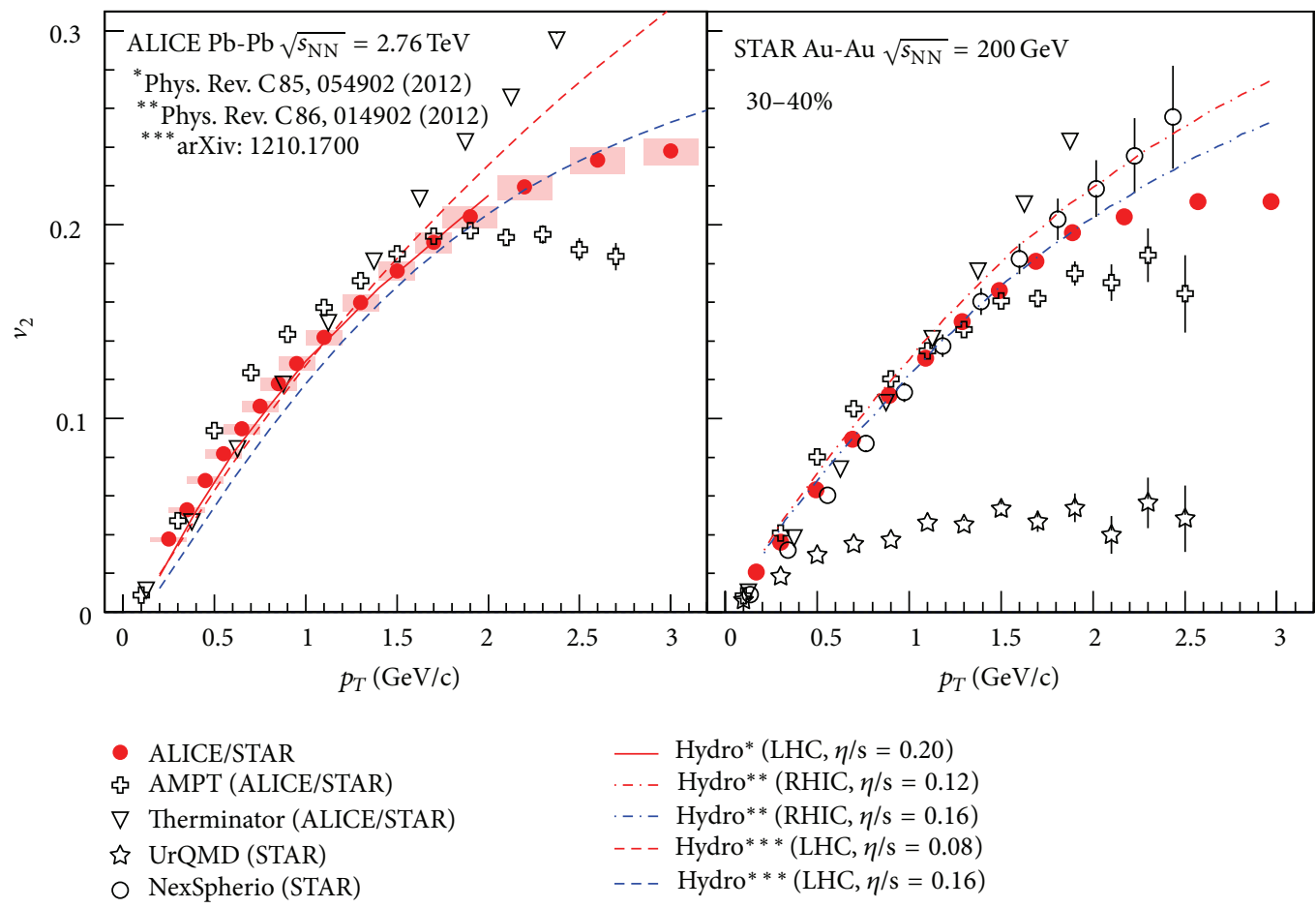

FIGURE 19: (Color online) The azimuthal anisotropy parameter $v_{2}$, measured in noncentral heavy-ion collisions at midrapidity for RHIC and LHC energies. For comparison, shown are the various theoretical calculations based on hydrodynamic and transport approaches (see text for details).

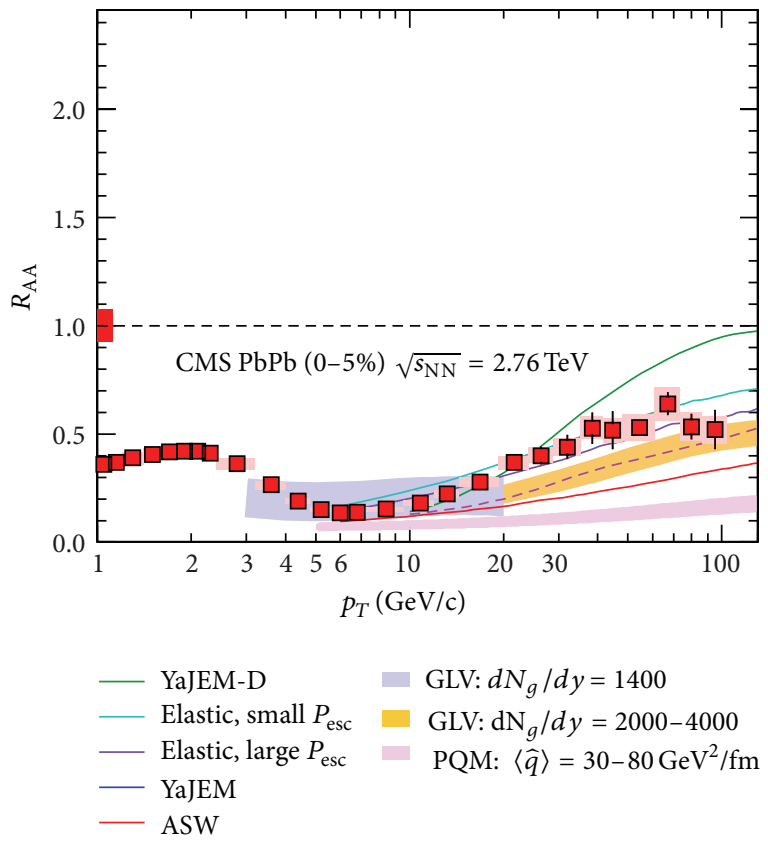

(a)

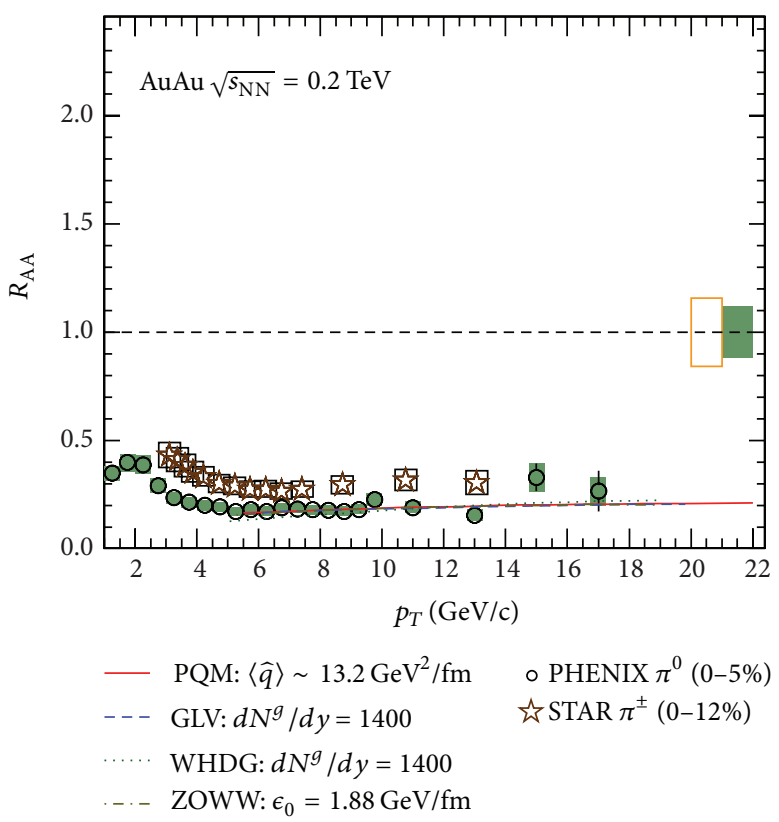

(b)

FIGURE 20: (Color online) Measurements of the nuclear modification factor $R_{\mathrm{AA}}$ in central heavy-ion collisions at two different center-of-mass energies, as a function of $p_{T}$, for pions $\left(\pi^{ \pm, 0}\right)[174,175]$ and charged hadrons [26, 27], compared to several theoretical predictions (see text). The error bars on the points are the statistical uncertainties, and the boxes around the data points are the systematic uncertainties. Additional absolute normalization uncertainties of order $5 \%$ to $10 \%$ are not plotted. The bands for several of the theoretical calculations represent their uncertainties. 
In this review, we showed that the first set of measurements made by the three LHC experiments within the heavy-ion programs, ALICE, ATLAS, and CMS, show a high degree of consistency. These measurements include centrality dependence of charged particle multiplicity, azimuthal anisotropy, and nuclear modification factor versus transverse momentum. Next, we discussed the comparison of various measurements made at RHIC and LHC energies. LHC measurements of $d N_{\mathrm{ch}} / d \eta$ clearly demonstrated the power law dependence of charged particle multiplicity on the beam energy. They also reconfirmed the observation at RHIC that particle production mechanism is not a simple superposition of several $p+p$ collisions. The values of $\left\langle m_{T}\right\rangle, \epsilon_{\mathrm{Bj}}$, freeze-out volume, decoupling time for hadrons, and $\left\langle v_{2}\right\rangle$ and $\langle\beta\rangle$ are larger at LHC energies compared to those at RHIC energies, even though the freeze-out temperatures are comparable. The value of the net-charge fluctuation measure is observed to rapidly approach towards a simple model-based calculation for QGP state. However, the sensitivity of this observable for a heavy-ion system as well as the lack of proper modeling of the heavy-ion system theoretically for such an observable needs careful consideration. The $v_{2}$ fluctuations as a function of centrality fraction have a similar value at both RHIC and LHC. This reflects their sensitivity to initial state effects. Just like at RHIC, the $R_{d \mathrm{Au}}$ and direct photon $R_{\mathrm{AA}}$ measurements experimentally demonstrated that the observed $R_{\mathrm{AA}}<1$ for charged hadrons is a final state effect; also at LHC, the $R_{p \mathrm{pb}}$, direct photon, and $W^{ \pm}$and $Z^{0} R_{\mathrm{AA}}$ measurements showed that the observed $R_{\mathrm{AA}}<1$ is indeed due to formation of a dense medium of colored charges in central heavy-ion collisions. All these conclusions were further validated by the comparison of several observables to corresponding model calculations. Further, it was found that the fluid at LHC shows a comparable degree of fluidity as that at RHIC. This is reflected by a small value of shear viscosity to entropy density ratio.

Measurements-related heavy quark production [166$168]$, dilepton production, jet-hadron correlations $[169,170]$, and higher-order azimuthal anisotropy $[171,172]$ which are now coming out of both RHIC and LHC experiments will provide a much more detailed characterization of the properties of the QCD matter formed in heavy-ion collisions.

\section{Acknowledgments}

The authors would like to thank F. Antinori, S. Gupta, D. Keane, A. K. Mohanty, Y. P. Viyogi, and N. Xu for reading the paper and for their helpful discussions and comments. Bedangadas Mohanty is supported by the DAE-SRC project fellowship for this work. Lokesh Kumar is partly supported by the DOE Grant DE-FG02-89ER40531 for carrying out this work.

\section{References}

[1] B. Mohanty, "Exploring the quantum chromodynamics landscape with high-energy nuclear collisions," New Journal of Physics, vol. 13, Article ID 065031, 2011.
[2] B. Mohanty, "QCD phase diagram: phase transition, critical point and fluctuations," Nuclear Physics A, vol. 830, no. 1-4, pp. 899C-907C, 2009.

[3] K. Fukushima and T. Hatsuda, "The phase diagram of dense QCD," Reports on Progress in Physics, vol. 74, no. 1, Article ID 014001, 2011.

[4] S. Gupta, X. Luo, B. Mohanty, H. G. Ritter, and N. Xu, "Scale for the phase diagram of quantum chromodynamics," Science, vol. 332, no. 6037, pp. 1525-1528, 2011.

[5] A. Bazavov, T. Bhattacharya, M. Cheng et al., "Chiral and deconfinement aspects of the QCD transition," Physical Review $D$, vol. 85, no. 5, Article ID 054503, 37 pages, 2012.

[6] S. Borsányi, Z. Fodor, C. Hoelbling et al., "Is there still any $T_{c}$ mystery in lattice QCD? Results with physical masses in the continuum limit III," Journal of High Energy Physics, vol. 2010, article 73, 2010.

[7] I. Arsene, I.G. Bearden, D. Beavis et al., "Quark-gluon plasma and color glass condensate at RHIC? The perspective from the BRAHMS experiment," Nuclear Physics A, vol. 757, no. 1-2, pp. $1-27,2005$.

[8] B. B. Back, M. D. Baker, M. Ballintijn et al., "The PHOBOS perspective on discoveries at RHIC," Nuclear Physics A, vol. 757, no. 1-2, pp. 28-101, 2005.

[9] J. Adams, M. M. Aggarwal, Z. Ahammed et al., "Experimental and theoretical challenges in the search for the quark-gluon plasma: the STAR collaboration's critical assessment of the evidence from RHIC collisions," Nuclear Physics A, vol. 757, no. 1-2, pp. 102-183, 2005.

[10] K. Adcox, S. S. Adlere, S. Afanasiev et al., "Formation of dense partonic matter in relativistic nucleus-nucleus collisions at RHIC: experimental evaluation by the PHENIX collaboration," Nuclear Physics A, vol. 757, no. 1-2, pp. 184-283, 2005.

[11] M. Gyulassy and L. McLerran, "New forms of QCD matter discovered at RHIC," Nuclear Physics A, vol. 750, no. 1, pp. 3063, 2005.

[12] B. Mohanty and STAR Collaboration, "STAR experiment results from the beam energy scan program at the RHIC," Journal of Physics G, vol. 38, no. 12, Article ID 124023, 2011.

[13] M. L. Miller, K. Reygers, S. J. Sanders, and P. Steinberg, "Glauber modeling in high-energy nuclear collisions," Annual Review of Nuclear and Particle Science, vol. 57, pp. 205-243, 2007.

[14] K. Aamodt, A. A. Quintana, D. Adamová et al., "Centrality dependence of the charged-particle multiplicity density at midrapidity in $\mathrm{Pb}-\mathrm{Pb}$ collisions at $\sqrt{s_{N N}}=2.76 \mathrm{TeV}$,' Physical Review Letters, vol. 106, no. 3, Article ID 032301, 10 pages, 2011.

[15] S. Chatrchyan, V. Khachatryan, A. M. Sirunyan et al., "Dependence on pseudorapidity and on centrality of charged hadron production in $\mathrm{PbPb}$ collisions at $\sqrt{s_{N N}}=2.76 \mathrm{TeV}$," Journal of High Energy Physics, vol. 2011, article 141, 2011.

[16] G. Aad, B. Abbott, J. Abdallah et al., "Measurement of the centrality dependence of the charged particle pseudorapidity distribution in lead-lead collisions at $\sqrt{s_{N N}}=2.76 \mathrm{TeV}$ with the ATLAS detector," Physics Letters B, vol. 710, no. 3, pp. 363-382, 2012.

[17] S. Chatrchyan, V. Khachatryan, A. M. Sirunyan et al., "Azimuthal anisotropy of charged particles at high transverse momenta in $\mathrm{Pb}-\mathrm{Pb}$ collisions at $\sqrt{s_{N N}}=2.76 \mathrm{TeV}$," Physical Review Letters, vol. 109, no. 2, Article ID 022301, 15 pages, 2012.

[18] S. Chatrchyan, V. Khachatryan, A. M. Sirunyan et al., "Measurement of the azimuthal anisotropy of neutral pions in $\mathrm{Pb}-\mathrm{Pb}$ collisions at $\sqrt{s_{N N}}=2.76 \mathrm{TeV}$," Physical Review Letters, vol. 110, no. 4, Article ID 042301, 15 pages, 2013. 
[19] S. Chatrchyan, V. Khachatryan, A. M. Sirunyan et al., "Measurement of the elliptic anisotropy of charged particles produced in $\mathrm{PbPb}$ collisions at $\sqrt{s_{N N}}=2.76 \mathrm{TeV}$," Physical Review C, vol. 87, no. 1, Article ID 014902, 34 pages, 2013.

[20] S. Chatrchyan, V. Khachatryan, A. M. Sirunyan et al., "Centrality dependence of dihadron correlations and azimuthal anisotropy harmonics in $\mathrm{PbPb}$ collisions at $\sqrt{s_{N N}}=2.76 \mathrm{TeV}$," European Physical Journal C, vol. 72, Article ID 10052, 2012.

[21] G. Aad, B. Abbott, J. Abdallah et al., "Measurement of the pseudorapidity and transverse momentum dependence of the elliptic flow of charged particles in lead-lead collisions at $\sqrt{s_{N N}}=2.76 \mathrm{TeV}$ with the ATLAS detector," Physics Letters $B$, vol. 707, no. 3-4, pp. 330-348, 2012.

[22] G. Aad, B. Abbott, J. Abdallah et al., "Measurement of the distributions of event-by-event flow harmonics in lead-lead collisions at $\sqrt{s_{N N}}=2.76 \mathrm{TeV}$ with the ATLAS detector at the LHC," http://arxiv.org/abs/1305.2942.

[23] G. Aad, B. Abbott, J. Abdallah et al., "Measurement of the azimuthal anisotropy for charged particle production in $\sqrt{s_{N N}}=2.76 \mathrm{TeV}$ lead-lead collisions with the ATLAS detector," Physical Review C, vol. 86, no. 1, Article ID 014907, 41 pages, 2012.

[24] J. Jia and ATLAS Collaboration, "Measurement of event plane correlations in $\mathrm{Pb}-\mathrm{Pb}$ collisions at $\sqrt{s_{N N}}=2.76 \mathrm{TeV}$ with the ATLAS detector," Nuclear Physics A, vol. 910-911, pp. 276-280, 2013.

[25] K. Aamodt, B. Abelev, A. A. Quintana et al., "Elliptic flow of charged particles in $\mathrm{Pb}-\mathrm{Pb}$ collisions at $\sqrt{s_{N N}}=2.76 \mathrm{TeV}$," Physical Review Letters, vol. 105, no. 25, Article ID 252302, 11 pages, 2010.

[26] K. Aamodt, B. Abelev, A. A. Quintana et al., "Suppression of charged particle production at large transverse momentum in central Pb-Pb collisions at $\sqrt{s_{N N}}=2.76 \mathrm{TeV}$,' Physics Letters $B$, vol. 696, no. 1-2, pp. 30-39, 2011.

[27] S. Chatrchyan, V. Khachatryan, A. M. Sirunyan et al., "Study of high-pT charged particle suppression in $\mathrm{PbPb}$ compared to $\mathrm{pp}$ collisions at $\sqrt{s_{N N}}=2.76 \mathrm{TeV}$,' European Physical Journal C, vol. 72, article 1945, 2012.

[28] A. N. Mishra and R. Sahoo, "Towards a universality in particle production in heavy-ion collisions," http://arxiv.org/abs/ 1304.2113.

[29] B. B. Back, M. D. Baker, M. Ballintijn et al., "Pseudorapidity distribution of charged particles in $d+$ Au collisions at $\sqrt{s_{N N}}=$ 200 GeV,' Physical Review Letters, vol. 93, no. 8, Article ID 082301, 5 pages, 2004.

[30] M. C. Abreu, B. Alessandro, C. Alexa et al., "Pseudorapidity distributions of charged particles as a function of centrality in $\mathrm{Pb}-\mathrm{Pb}$ collisions at 158 and $40 \mathrm{GeV}$ per nucleon incident energy," Physics Letters B, vol. 530, no. 1-4, pp. 33-42, 2002.

[31] C. Adler, Z. Ahammed, C. Allgower et al., "Multiplicity distribution and spectra of negatively charged hadrons in $A u+A u$ collisions at $\sqrt{s_{N N}}=130 \mathrm{GeV}$,' Physical Review Letters, vol. 87, no. 11, Article ID 112303, 5 pages, 2001.

[32] I. G. Bearden, D. Beavis, C. Besliu et al., "Charged particle densities from $\mathrm{Au}+\mathrm{Au}$ collisions at $\sqrt{s_{N N}}=130 \mathrm{GeV}$," Physics Letters B, vol. 523, no. 3-4, pp. 227-233, 2001.

[33] I. G. Bearden, D. Beavis, C. Besliu et al., "Pseudorapidity distributions of charged particles from $\mathrm{Au}+\mathrm{Au}$ collisions at the maximum RHIC energy, $\sqrt{s_{N N}}=200 \mathrm{GeV}$," Physical Review Letters, vol. 88, no. 20, Article ID 202301, 4 pages, 2002.

[34] K. Adcox, S. S. Adler, N. N. Ajitanand et al., "Centrality dependence of charged particle multiplicity in Au-Au collisions at $\sqrt{s_{N N}}=130 \mathrm{GeV}$,' Physical Review Letters, vol. 86, no. 16, pp. 3500-3505, 2001.

[35] B. B. Back, M. D. Baker, D. S. Barton et al., "Charged-particle multiplicity near midrapidity in central $\mathrm{Au}+\mathrm{Au}$ Collisions at $\sqrt{s_{N N}}=130 \mathrm{GeV}$," Physical Review Letters, vol. 85, no. 15, pp. 3100-3104, 2000.

[36] B. B. Back, M. D. Baker, D. S. Barton et al., "Significance of the fragmentation region in ultrarelativistic heavy-ion collisions," Physical Review Letters, vol. 91, no. 5, Article ID 052303, 4 pages, 2003.

[37] W. Thome, K. Eggert, K. Giboni et al., "Charged particle multiplicity distributions in pp collisions at ISR energies," Nuclear Physics B, vol. 129, no. 3, pp. 365-389, 1977.

[38] C. Albajar, M. G. Albrow, O. C. Allkofer et al., "A study of the general characteristics of proton-antiproton collisions at $\sqrt{s}=$ 0.2 to 0.9 TeV," Nuclear Physics B, vol. 335, no. 2, pp. 261-287, 1990.

[39] K. Alpgard, R. E. Ansorge, B. Åsman et al., "Particle multiplicities in $\bar{p} p$ interactions at $\sqrt{s}=540 \mathrm{GeV}$," Physics Letters B, vol. 121, no. 2-3, pp. 209-215, 1983.

[40] G. Alner, R. E. Ansorge, B. Åsman et al., "Scaling of pseudorapidity distributions at c.m. energies up to $0.9 \mathrm{TeV}$," Zeitschrift für Physik C, vol. 33, no. 1, pp. 1-6, 1986.

[41] T. Alber and NA35 Collaboration, "Charged particle production in proton-, deuteron-, oxygen- and sulphur-nucleus collisions at $200 \mathrm{GeV}$ per nucleon," The European Physical Journal C, vol. 2, no. 4, pp. 643-659, 1998.

[42] F. Abe, D. Amidei, G. Apollinari et al., "Pseudorapidity distributions of charged particles produced in $\bar{p} p$ interactions as $\sqrt{s}=630$ and $1800 \mathrm{GeV}$," Physical Review D, vol. 41, no. 7, pp. 2330-2333, 1990.

[43] K. Aamodt, B. Abelev, A. Quintana et al., "Charged-particle multiplicity measurement in proton-proton collisions at $\sqrt{s}=$ $7 \mathrm{TeV}$ with ALICE at LHC," The European Physical Journal C, vol. 68, no. 3-4, pp. 345-354, 2010.

[44] V. Khachatryan, M. Sirunyan, A. Tumasyan et al., "Transverse momentum and pseudorapidity distributions of charged hadrons in pp collisions at $\sqrt{s}=0.9$ and $2.36 \mathrm{TeV}$,' Journal of High Energy Physics, vol. 1002, article 41, 2010.

[45] W. Thome, K. Eggert, K. Giboni et al., "Charged particle multiplicity distributions in pp collisions at ISR energies," Nuclear Physics B, vol. 129, no. 3, pp. 365-389, 1977.

[46] K. Aamodt, N. Abel, U. Abeysekara et al., "Charged-particle multiplicity measurement in proton-proton collisions at $\sqrt{s}=$ 0.9 and $2.36 \mathrm{TeV}$ with ALICE at LHC," European Physical Journal C, vol. 68, no. 1-2, pp. 89-108, 2010.

[47] B. Alver, B. B. Back, M. D. Baker et al., "Charged-particle multiplicity and pseudorapidity distributions measured with the PHOBOS detector in $\mathrm{Au}+\mathrm{Au}, \mathrm{Cu}+\mathrm{Cu}, \mathrm{d}+\mathrm{Au}$, and $\mathrm{p}+\mathrm{p}$ collisions at ultrarelativistic energies," Physical Review C, vol. 83, no. 2, Article ID 024913, 24 pages, 2011.

[48] B. Abelev, J. Adam, D. Adamová et al., "Pseudorapidity density of charged particles in $\mathrm{p}+\mathrm{Pb}$ collisions at $\sqrt{s_{N N}}=5.02 \mathrm{TeV}$," Physical Review Letters, vol. 110, no. 3, Article ID 032301, 10 pages, 2013.

[49] T. Alber and NA35 Collaboration, "Charged particle production in proton-, deuteron-, oxygen- and sulphur-nucleus collisions at $200 \mathrm{GeV}$ per nucleon," The European Physical Journal C, vol. 2, no. 4, pp. 643-659, 1998.

[50] L. Ahle, Y. Akibad, K. Ashktorab et al., "Excitation function of $\mathrm{K}+$ and $\pi+$ production in $\mathrm{Au}+\mathrm{Au}$ reactions at 2-10 AGeV," Physics Letters B, vol. 476, no. 1-2, pp. 1-8, 2000. 
[51] J. Barrette, R. Bellwied, S. Bennett et al., "Proton and pion production in Au+Au collisions at 10.8A GeV/c," Physical Review C, vol. 62, no. 2, Article ID 024901, 8 pages, 2000.

[52] J. T. Mitchell and PHENIX Collaboration, "The RHIC beam energy scan program: results from the PHENIX experiment," Nuclear Physics A, vol. 904-905, pp. 903c-906c, 2013.

[53] C. Alt, T. Anticic, B. Baatar et al., "Pion and kaon production in central $\mathrm{Pb}+\mathrm{Pb}$ collisions at $20 \mathrm{~A}$ and $30 \mathrm{~A} \mathrm{GeV}$ : evidence for the onset of deconfinement," Physical Review C, vol. 77, no. 2, Article ID 024903, 10 pages, 2008.

[54] B. I. Abelev, M. M. Aggarwal, Z. Ahammed et al., "Identified particle production, azimuthal anisotropy, and interferometry measurements in $\mathrm{Au}+\mathrm{Au}$ collisions at $\sqrt{s_{N N}}=9.2 \mathrm{GeV}$," Physical Review C, vol. 81, no. 2, Article ID 024911, 19 pages, 2010.

[55] B. Abelev, J. Adam, D. Adamová et al., "Pion, kaon, and proton production in central $\mathrm{Pb}-\mathrm{Pb}$ collisions at $\sqrt{s_{N N}}=2.76 \mathrm{TeV}$," Physical Review Letters, vol. 109, no. 25, Article ID 252301, 11 pages, 2012.

[56] B. Mohanty, J. E. Alam, S. Sarkar, T. K. Nayak, and B. K. Nandi, "Indication of a coexisting phase of quarks and hadrons in nucleus-nucleus collisions," Physical Review C, vol. 68, no. 2, Article ID 021901, 4 pages, 2003.

[57] L. Van Hove, "Multiplicity dependence of pt spectrum as a possible signal for a phase transition in hadronic collisions," Physics Letters B, vol. 118, no. 1-3, pp. 138-140, 1982.

[58] J. D. Bjorken, "Highly relativistic nucleus-nucleus collisions: the central rapidity region," Physical Review D, vol. 27, no. 1, pp. 140151, 1983.

[59] C. Loizides, "Charged-particle multiplicity and transverse energy in $\mathrm{Pb}-\mathrm{Pb}$ collisions at $\sqrt{s_{N N}}=2.76 \mathrm{TeV}$ with ALICE," Journal of Physics G, vol. 38, no. 12, Article ID 124040, 2011.

[60] S. Chatrchyan, V. Khachatryan, A. M. Sirunyan et al., "Measurement of the pseudorapidity and centrality dependence of the transverse energy density in $\mathrm{Pb}-\mathrm{Pb}$ collisions at $\sqrt{s_{N N}}=$ 2.76 TeV," Physical Review Letters, vol. 109, no. 15, Article ID 152303, 16 pages, 2012.

[61] J. T. Mitchell and PHENIX Collaboration, "The RHIC beam energy scan program: results from the PHENIX experiment," http://arxiv.org/abs/1211.6139.

[62] S. S. Adler, S. Afanasiev, C. Aidala et al., "Systematic studies of the centrality and $\sqrt{s_{N N}}$ dependence of the $\mathrm{dET} / \mathrm{d} \eta$ and $\mathrm{dNch} / \mathrm{d} \eta$ in heavy ion collisions at midrapidity," Physical Review C, vol. 71, no. 3, Article ID 034908, 25 pages, 2005, Erratum in Physical Review C, vol. 71, Article ID 049901, 2005.

[63] K. Adcox, S. S. Adler, N. N. Ajitanand et al., "Measurement of the midrapidity transverse energy distribution from $\sqrt{s_{N N}}=$ $130 \mathrm{GeV}$ Au+Au collisions at RHIC," Physical Review Letters, vol. 87, no. 5, Article ID 052301, 6 pages, 2001.

[64] M. M. Aggarwal, A. Agnihotri, Z. Ahammed et al., "Scaling of particle and transverse energy production in ${ }^{208} \mathrm{~Pb}+{ }^{208} \mathrm{~Pb}$ collisions at 158.A GeV," The European Physical Journal C, vol. 18, no. 4, pp. 651-663, 2001.

[65] G. F. Bertsch, "Pion interferometry as a probe of the plasma," Nuclear Physics A, vol. 498, pp. 173-179, 1989.

[66] S. Pratt, "Pion interferometry of quark-gluon plasma," Physical Review D, vol. 33, no. 5, pp. 1314-1327, 1986.

[67] D. Teaney, "Effect of shear viscosity on spectra, elliptic flow, and Hanbury Brown-Twiss radii," Physical Review C, vol. 68, no. 3, Article ID 034913, 11 pages, 2003.

[68] M. A. Lisa, N. N. Ajitanand, J. M. Alexander et al., "Bombarding energy dependence of $\pi$-interferometry at the Brookhaven
AGS," Physical Review Letters, vol. 84, no. 13, pp. 2798-2802, 2000.

[69] C. Alt, T. Anticic, B. Baatar et al., "Bose-Einstein correlations of $\pi$ - $\pi$-pairs in central $\mathrm{Pb}+\mathrm{Pb}$ collisions at $20 \mathrm{~A}, 30 \mathrm{~A}, 40 \mathrm{~A}, 80 \mathrm{~A}$, and 158A GeV," Physical Review C, vol. 77, no. 6, Article ID 064908, 20 pages, 2008.

[70] D. Adamova, G. Agakichievb, H. Appelshäuser et al., "Beam energy and centrality dependence of two-pion Bose-Einstein correlations at SPS energies," Nuclear Physics A, vol. 714, no. 1-2, pp. 124-144, 2003.

[71] B. I. Abelev, M. M. Aggarwal, Z. Ahammed et al., "Pion interferometry in $\mathrm{Au}+\mathrm{Au}$ and $\mathrm{Cu}+\mathrm{Cu}$ collisions at $\sqrt{s_{N N}}=$ 62.4 and $200 \mathrm{GeV}$," Physical Review C, vol. 80, no. 2, Article ID 024905, 12 pages, 2009.

[72] B. B. Back, M. D. Baker, M. Ballintijn et al., "Transverse momentum and rapidity dependence of Hanbury-Brown-Twiss correlations in Au+Au collisions at $\sqrt{s_{N N}}=62.4$ and $200 \mathrm{GeV}$," Physical Review C, vol. 73, no. 3, Article ID 031901, 5 pages, 2006.

[73] K. Aamodt, A. A. Quintana, D. Adamová et al., “Twopion Bose-Einstein correlations in central $\mathrm{Pb}-\mathrm{Pb}$ collisions at $\sqrt{s_{N N}}=2.76$ TeV," Physics Letters B, vol. 696, no. 4, pp. 328-337, 2011.

[74] W. Reisdorfa, D. Besta, A. Gobbi et al., "Central collisions of Au on Au at 150, 250 and $400 \mathrm{~A} \cdot \mathrm{MeV}$," Nuclear Physics A, vol. 612, pp. 493-556, 1997.

[75] M. A. Lisa, S. Albergo, F. Bieser et al., "Radial flow in $\mathrm{Au}+\mathrm{Au}$ collisions at $E=(0.25-1.15)$ AGeV," Physical Review Letters, vol. 75 , pp. 2662-2665, 1995.

[76] C. Muntz and E802 Collaboration, "Recent results from E866 at BNL," http://arxiv.org/abs/nucl-ex/9806002.

[77] H. Appelshäuser, J. Bächler, S. J. Bailey et al., "Hadronic expansion dynamics in central $\mathrm{Pb}+\mathrm{Pb}$ collisions at $158 \mathrm{GeV}$ per nucleon," European Physical Journal C, vol. 2, pp. 661-670, 1998.

[78] A. Andronic, P. Braun-Munzinger, and J. Stachel, "Hadron production in central nucleus-nucleus collisions at chemical freeze-out," Nuclear Physics A, vol. 772, no. 3-4, pp. 167-199, 2006.

[79] A. Andronic, P. Braun-Munzinger, and J. Stachel, "Thermal hadron production in relativistic nuclear collisions: the hadron mass spectrum, the horn, and the QCD phase transition," Physics Letters B, vol. 673, no. 2, pp. 142-145, 2009, Erratum in Physics Letters B, vol. 678, p. 516, 2009.

[80] J. Cleymans, H. Oeschler, and K. Redlich, "Particle ratios at SPS, AGS and SIS," Journal of Physics G, vol. 25, no. 2, p. 281, 1999.

[81] J. Cleymans, D. Elliott, A. Keranen, and E. Suhonen, “Thermal model analysis of particle ratios in $\mathrm{Ni}+\mathrm{Ni}$ experiments using exact strangeness conservation," Physical Review C, vol. 57, no. 6, pp. 3319-3323, 1998.

[82] P. Braun-Munzinger, J. Stachel, J. P. Wessels, and N. Xu, "Thermal equilibration and expansion in nucleus-nucleus collisions at the AGS," Physics Letters B, vol. 344, no. 1-4, pp. 43-48, 1995.

[83] P. Braun-Munzinger, I. Heppe, and J. Stachel, "Chemical equilibration in $\mathrm{Pb}+\mathrm{Pb}$ collisions at the SPS," Physics Letters B, vol. 465, no. 1-4, pp. 15-20, 1999.

[84] F. Becattini and G. Pettini, "Strange quark production in a statistical effective model," Physical Review C, vol. 67, no. 1, Article ID 015205, 12 pages, 2003.

[85] F. Becattini, J. Cleymans, A. Keranen, E. Suhonen, and K. Redlich, "Features of particle multiplicities and strangeness production in central heavy ion collisions between 1.7A and 158A GeV/c," Physical Review C, vol. 64, no. 2, Article ID 024901, 9 pages, 2001. 
[86] L. Milano and ALICE Collaboration, "Identified charged hadron production in $\mathrm{Pb}-\mathrm{Pb}$ collisions at the LHC with the ALICE Experiment," Nuclear Physics A, vol. 904-905, pp. 531c534c, 2013.

[87] J. Steinheimer, J. Aichelin, and M. Bleicher, "Nonthermal $\mathrm{p} / \pi$ ratio at $\mathrm{LHC}$ as a consequence of hadronic final state interactions," Physical Review Letters, vol. 110, no. 4, Article ID 042501, 4 pages, 2013.

[88] C. Pruneau, S. Gavin, and S. Voloshin, "Methods for the study of particle production fluctuations," Physical Review C, vol. 66, no. 4, Article ID 044904, 12 pages, 2002.

[89] H. Sako, H. Appelshäuser, and CERES/NA45 Collaboration, "vent-by-event fluctuations at 40,80 and $158 \mathrm{~A} \mathrm{GeV} / \mathrm{c}$ in $\mathrm{Pb}+\mathrm{Au}$ collisions," Journal of Physics G, vol. 30, no. 8, Article ID S1371, 2004.

[90] B. I. Abelev, M. M. Aggarwal, Z. Ahammed et al., "Beamenergy and system-size dependence of dynamical net charge fluctuations," Physical Review C, vol. 79, no. 2, Article ID 024906, 14 pages, 2009.

[91] B. Abelev, J. Adam, D. Adamová et al., "Net-charge fluctuations in $\mathrm{Pb}-\mathrm{Pb}$ collisions at $\sqrt{s_{N N}}=2.76 \mathrm{TeV}$," Physical Review Letters, vol. 110, no. 15, Article ID 152301, 11 pages, 2013.

[92] S. Jeon and V. Koch, "Charged particle ratio fluctuation as a signal for quark-gluon plasma," Physical Review Letters, vol. 85, no. 10, pp. 2076-2079, 2000.

[93] J. Y. Ollitrault, "Anisotropy as a signature of transverse collective flow," Physical Review D, vol. 46, no. 1, pp. 229-245, 1992.

[94] A. Andronic, V. Barret, Z. Basrak et al., "Excitation function of elliptic flow in $\mathrm{Au}+\mathrm{Au}$ collisions and the nuclear matter equation of state," Physics Letters B, vol. 612, no. 3-4, pp. 173180, 2005.

[95] N. Bastidm, A. Andronic, V. Barret et al., "First analysis of anisotropic flow with Lee-Yang zeros," Physical Review C, vol. 72, no. 1, Article ID 011901, 5 pages, 2005.

[96] ATLAS Collaboration, "Search for supersymmetry in events with at least one photon, one lepton, and large missing transverse momentum in proton-proton collision at a center-ofmass energy of $7 \mathrm{TeV}$ with the ATLAS detector," ATLAS-CONF2012-117.

[97] C. Pinkenburg, N. N. Ajitanand, J. M. Alexander et al., "Elliptic flow: transition from out-of-plane to in-plane emission in $\mathrm{Au}+\mathrm{Au}$ collisions," Physical Review Letters, vol. 83, no. 7, pp. 1295-1298, 1999.

[98] D. Adamova and CERES collaboration, "New results from CERES," Nuclear Physics A, vol. 698, no. 1-4, pp. 253-260, 2002.

[99] C. Alt, T. Anticic, B. Baatar et al., "Directed and elliptic flow of charged pions and protons in $\mathrm{Pb}+\mathrm{Pb}$ collisions at $40 \mathrm{~A}$ and 158A GeV," Physical Review C, vol. 68, no. 3, Article ID 034903, 32 pages, 2003.

[100] J. Adams, M. M. Aggarwal, Z. Ahammed et al., "Azimuthal anisotropy in $\mathrm{Au}+\mathrm{Au}$ collisions at $\sqrt{s_{N N}}=200 \mathrm{GeV}$," Physical Review C, vol. 72, no. 1, Article ID 014904, 23 pages, 2005.

[101] B. B. Back, M. D. Baker, M. Ballintijn et al., "Energy dependence of elliptic flow over a large pseudorapidity range in $\mathrm{Au}+\mathrm{Au}$ collisions at the BNL relativistic heavy ion collider," Physical Review Letters, vol. 94, no. 12, Article ID 122303, 4 pages, 2005.

[102] S. Afanasiev, C. Aidala, N. N. Ajitanand et al., "Systematic studies of elliptic flow measurements in $\mathrm{Au}+\mathrm{Au}$ collisions at $\sqrt{s_{N N}}=200 \mathrm{GeV}$," Physical Review C, vol. 80 , no. 2, Article ID 024909, 25 pages, 2009.

[103] ATLAS Collaboration, ATLAS-CONF-2012-117.
[104] A. Adare, S. Afanasiev, C. Aidala et al., "Measurements of higher order flow harmonics in Au+Au collisions at $\sqrt{s_{N N}}=200 \mathrm{GeV}$," Physical Review Letters, vol. 107, no. 25, Article ID 252301, 7 pages, 2011.

[105] K. Aamodt, B. Abelev, A. A. Quintana et al., "Higher harmonic anisotropic flow measurements of charged particles in $\mathrm{Pb}-\mathrm{Pb}$ collisions at $\sqrt{s_{N N}}=2.76 \mathrm{TeV}$," Physical Review Letters, vol. 107, no. 3, Article ID 032301, 10 pages, 2011.

[106] A. Adare, S. Afanasiev, C. Aidala et al., "Deviation from quark number scaling of the anisotropy parameter v2 of pions, kaons, and protons in $\mathrm{Au}+\mathrm{Au}$ collisions at $\sqrt{s_{N N}}=200 \mathrm{GeV}$,' Physical Review C, vol. 85, no. 6, Article ID 064914, 14 pages, 2012.

[107] B. Abelev, J. Adam, D. Adamová et al., "Anisotropic flow of charged hadrons, pions and (anti-)protons measured at high transverse momentum in $\mathrm{Pb}-\mathrm{Pb}$ collisions at $\sqrt{s_{N N}}=2.76 \mathrm{TeV}$," Physics Letters B, vol. 719, no. 1-3, pp. 18-28, 2013.

[108] B. Müller, R. J. Fries, and S. A. Bass, “Thermal recombination: beyond the valence quark approximation," Physics Letters B, vol. 618, no. 1-4, pp. 77-83, 2005.

[109] V. Greco and C. M. Ko, "Effect of space-momentum correlations on the constituent quark number scaling of hadron elliptic flows," http://arxiv.org/abs/nucl-th/0505061.

[110] G. Agakishiev, M. M. Aggarwal, Z. Ahammed et al., "Energy and system-size dependence of two- and four-particle v2 measurements in heavy-ion collisions at $\sqrt{s_{N N}}=62.4$ and $200 \mathrm{GeV}$ and their implications on flow fluctuations and nonflow," Physical Review C, vol. 86, no. 1, Article ID 014904, 15 pages, 2012.

[111] S. A. Voloshin, A. M. Poskanzer, A. Tang, and G. Wang, "Elliptic flow in the Gaussian model of eccentricity fluctuations," Physics Letters B, vol. 659, no. 3, pp. 537-541, 2008.

[112] S. Voloshin and Y. Zhang, "Flow study in relativistic nuclear collisions by Fourier expansion of Azimuthal particle distributions," Zeitschrift für Physik C, vol. 70, no. 4, pp. 665-671, 1996.

[113] S. A. Voloshin, A. M. Poskanzer, and R. Snellings, "Collective phenomena in non-central nuclear collisions," http://arxiv.org/ abs/0809.2949.

[114] G. Aad and ATLAS Collaboration, "Measurement of the distributions of event-by-event flow harmonics in lead-lead collisions at $\sqrt{s_{N N}}=2.76 \mathrm{TeV}$ with the ATLAS detector at the LHC," http://arxiv.org/abs/1305.2942.

[115] J. Adams, C. Adler, M. M. Aggarwal et al., "Evidence from d+Au measurements for final-state suppression of high-pT hadrons in $\mathrm{Au}+\mathrm{Au}$ collisions at RHIC," Physical Review Letters, vol. 91, no. 7, Article ID 072304, 6 pages, 2003.

[116] B. Abelev, J. Adam, D. Adamová et al., “Transverse momentum distribution and nuclear modification factor of charged particles in $\mathrm{p}+\mathrm{Pb}$ collisions at $\sqrt{s_{N N}}=5.02 \mathrm{TeV}$," Physical Review Letters, vol. 110, no. 8, Article ID 082302, 11 pages, 2013.

[117] D. Antreasyan, J. W. Cronin, H. J. Frisch et al., "Production of kaons, protons, and antiprotons with large transverse momentum in p-p and p-d collisions at 200,300, and $400 \mathrm{GeV}$,' Physical Review Letters, vol. 38, no. 3, pp. 115-117, 1977.

[118] D. Antreasyan, J. W. Cronin, H. J. Frisch et al., "Production of $\pi+$ and $\pi$ - at large transverse momentum in p-p and p-d collisions at 200, 300, and 400 GeV," Physical Review Letters, vol. 38, no. 3, pp. 112-114, 1977.

[119] S. S. Adler, S. Afanasiev, C. Aidala et al., "Centrality dependence of direct photon production in $\sqrt{s_{N N}}=200 \mathrm{GeV} \mathrm{Au+Au}$ collisions," Physical Review Letters, vol. 94, no. 23, Article ID 232301, 6 pages, 2005. 
[120] S. Chatrchyan, V. Khachatryan, A.M. Sirunyan et al., "Measurement of isolated photon production in $\mathrm{pp}$ and $\mathrm{PbPb}$ collisions at $\sqrt{s_{N N}}=2.76$ TeV," Physics Letters B, vol. 710, no. 2, pp. 256-277, 2012.

[121] S. Chatrchyan, V. Khachatryan, A.M. Sirunyan et al., "Study of $\mathrm{W}$ boson production in $\mathrm{PbPb}$ and pp collisions at $\sqrt{s_{N N}}=$ 2.76 TeV," Physics Letters B, vol. 715, no. 1-3, pp. 66-87, 2012.

[122] S. Chatrchyan, V. Khachatryan, A. M. Sirunyan et al., "Study of $\mathrm{Z}$ boson production in $\mathrm{PbPb}$ collisions at $\sqrt{s_{N N}}=2.76 \mathrm{TeV}$," Physical Review Letters, vol. 106, no. 21, Article ID 212301, 14 pages, 2011.

[123] W. Busza, "Trends in multiparticle production and some "predictions" for $\mathrm{pp}$ and $\mathrm{PbPb}$ collisions at LHC," Journal of Physics G, vol. 35, no. 4, Article ID 044040, 2008.

[124] S. Barshay and G. Kreyerhoff, "Related power-law growth of particle multiplicities near midrapidity in central $\mathrm{Au}+\mathrm{Au}$ collisions and in $\bar{p}$-p collisions," Nuclear Physics A, vol. 697, no. 1-2, pp. 563-568, 2002, Erratum in Nuclear Physics A, vol. 703, p. 891, 2002.

[125] W. T. Deng, X. N. Wang, and R. Xu, "Hadron production in $\mathrm{p}+\mathrm{p}$, $\mathrm{p}+\mathrm{Pb}$, and $\mathrm{Pb}+\mathrm{Pb}$ collisions with the hijing 2.0 model at energies available at the CERN large hadron collider," Physical Review C, vol. 83, no. 1, Article ID 014915, 9 pages, 2011.

[126] F. W. Bopp, R. Engel, J. Ranft, and S. Roesler, "Inclusive distributions at the LHC as predicted from the DPMJET-III model with chain fusion," http://arxiv.org/abs/0706.3875.

[127] M. Mitrovski, T. Schuster, G. Graf, H. Petersen, and M. Bleicher, "Charged-particle (pseudo-)rapidity distributions in $\mathrm{p}+\bar{p} / \mathrm{p}+\mathrm{p}$ and $\mathrm{Pb}+\mathrm{Pb} / \mathrm{Au}+\mathrm{Au}$ collisions from UrQMD calculations at energies available at the CERN super proton synchrotron to the large hadron collider," Physical Review C, vol. 79, no. 4, Article ID 044901, 6 pages, 2009.

[128] S. Jeon and J. I. Kapusta, "Interpretation of the first data on central Au+Au collisions at $\sqrt{s}=56 \mathrm{~A}$ and 130A GeV," Physical Review C, vol. 63, no. 1, Article ID 011901, 3 pages, 2001.

[129] J. L. Albacete, "CGC and initial state effects in heavy ion collisions," Journal of Physics: Conference Series, vol. 270, no. 1, Article ID 012052, 2011.

[130] E. Levin and A. H. Rezaeian, "Hadron multiplicity in pp and AA collisions at LHC from the color glass condensate," Physical Review D, vol. 82, no. 5, Article ID 054003, 6 pages, 2010.

[131] D. Kharzeev, E. Levin, and M. Nardi, "Color glass condensate at the LHC: hadron multiplicities in pp, pA and AA collisions," Nuclear Physics A, vol. 747, no. 2-4, pp. 609-629, 2005.

[132] D. Kharzeev, E. Levin, and M. Nardi, "Hadron multiplicities at the LHC," http://arxiv.org/abs/0707.0811.

[133] N. Armesto, C. A. Salgado, and U. A. Wiedemann, "Relating high-energy Lepton-Hadron, proton-nucleus, and nucleusnucleus collisions through geometric scaling," Physical Review Letters, vol. 94, no. 2, Article ID 022002, 4 pages, 2005.

[134] K. J. Eskola, P. V. Ruuskanen, S. S. Räsänen, and K. Tuominen, "Multiplicities and transverse energies in central AA collisions at RHIC and LHC from pQCD, saturation and hydrodynamics," Nuclear Physics A, vol. 696, no. 3-4, pp. 715-728, 2001.

[135] K. J. Eskola, K. Kajantie, and K. Tuominen, "Heavy-ion collision multiplicities and gluon distribution functions," Nuclear Physics A, vol. 700, no. 1-2, pp. 509-522, 2002.

[136] P. Bozek, M. Chojnacki, W. Florkowski, and B. Tomášik, "Hydrodynamic predictions for $\mathrm{Pb}+\mathrm{Pb}$ collisions at $\sqrt{s_{N N}}=$ 2.76 TeV," Physics Letters B, vol. 694, no. 3, pp. 238-241, 2010.
[137] E. K. G. Sarkisyan and A. S. Sakharov, "Relating multihadron production in hadronic and nuclear collisions," European Physical Journal C, vol. 70, no. 3, pp. 533-541, 2010.

[138] E. K. G. Sarkisyan and A. S. Sakharov, "Multihadron production features in different reactions," AIP Conference Proceedings, vol. 828, pp. 35-41, 2006.

[139] T. J. Humanic, "Predictions of hadronic observables in $\mathrm{Pb}+\mathrm{Pb}$ collisions at $\sqrt{s_{N N}}=2.76 \mathrm{TeV}$ from a hadronic rescattering model," http://arxiv.org/abs/1011.0378.

[140] A. Accardi, "Semi-hard scatterings at RHIC and LHC: initial conditions and charged multiplicities," http://arxiv.org/abs/ hep-ph/0104060.

[141] N. Armesto, C. Pajares, and D. Sousa, "Analysis of the first RHIC results in the string fusion model," Physics Letters B, vol. 527, no. 1-2, pp. 92-98, 2002.

[142] J. D. de Deus and R. Ugoccioni, "Particle densities in heavy ion collisions at high energy and the dual string model," Physics Letters B, vol. 491, no. 3-4, pp. 253-256, 2000.

[143] D. E. Kahana and S. H. Kahana, "Inclusive particle spectra at (56 and 130)A GeV," Physical Review C, vol. 63, no. 3, Article ID 031901, 4 pages, 2001.

[144] J. L. ALbacete and A. Dumitru, "A model for gluon production in heavy-ion collisions at the LHC with rcBK unintegrated gluon densities," http://arxiv.org/abs/1011.5161.

[145] P. Koch, B. Muller, and J. Rafelski, "Strangeness in relativistic heavy ion collisions," Physics Reports, vol. 142, no. 4, pp. 167262, 1986.

[146] M. Gaździcki and M. I. Gorenstein, "On the early stage of nucleus-nucleus collisions," Acta Physica Polonica B, vol. 30, p. 2705, 1999.

[147] I. Kuznetsova and J. Rafelski, "Non-equilibrium heavy-flavored hadron yields from chemical equilibrium strangeness-rich QGP," Journal of Physics G, vol. 35, no. 4, Article ID 044011, 2008.

[148] J. Cleymans, H. Oeschler, K. Redlich, and S. Wheaton, "The thermal model and the transition from baryonic to mesonic freeze-out," European Physical Journal A, vol. 29, no. 1, pp. 119$121,2006$.

[149] B. Tomasik and E.E. Kolomeitsev, "Strangeness production time and the $\mathrm{K}+/ \pi+$ horn," European Physical Journal $C$, vol. 49, no. 1, pp. 115-120, 2007.

[150] S. Chatterjee, R. M. Godbole, and S. Gupta, "Stabilizing hadron resonance gas models," Physical Review C, vol. 81, no. 4, Article ID 044907, 7 pages, 2010.

[151] C. Shen and U. Heinz, "Collision energy dependence of viscous hydrodynamic flow in relativistic heavy-ion collisions," Physical Review C, vol. 85, no. 5, Article ID 054902, 12 pages, 2012, Erratum in Physical Review C, vol. 86, Aritcle ID 049903, 2012.

[152] V. Roy, A. K. Chaudhuri, and B. Mohanty, "Comparison of results from a (2+1)-D relativistic viscous hydrodynamic model to elliptic and hexadecapole flow of charged hadrons measured in Au-Au collisions at $\sqrt{s_{N N}}=200 \mathrm{GeV}$," Physical Review C, vol. 86, no. 1, Article ID 014902, 9 pages, 2012.

[153] V. Roy, B. Mohanty, and A. K. Chaudhuri, "Elliptic and hexadecapole flow of charged hadrons in viscous hydrodynamics with Glauber and color glass condensate initial conditions for $\mathrm{Pb}-\mathrm{Pb}$ collision at $\sqrt{s_{N N}}=2.76 \mathrm{TeV}$,' Journal of Physics G, vol. 40, no. 6, Article ID 065103, 2013.

[154] J. Xu and C. M. Ko, "Pb-Pb collisions at $\sqrt{s_{N N}}=2.76 \mathrm{TeV}$ in a multiphase transport model," Physical Review C, vol. 83, no. 3, Article ID 034904, 5 pages, 2011. 
[155] M. Chojnacki, A. Kisiel, W. Florkowski, and W. Broniowski, "THERMINATOR 2: THERMal heavy IoN generATOR 2," Computer Physics Communications, vol. 183, no. 3, pp. 746-773, 2012.

[156] A. Kisiel, T. Tałuć, W. Broniowski, and W. Florkowski, “THERMINATOR: THERMal heavy-IoN generATOR," Computer Physics Communications, vol. 174, no. 8, pp. 669-687, 2006.

[157] S. A. Bass, M. Belkacem, M. Bleicher et al., "Microscopic models for ultrarelativistic heavy ion collisions," Progress in Particle and Nuclear Physics, vol. 41, pp. 255-369, 1998.

[158] M. Bleicher, E. Zabrodin, C. Spieles et al., "Relativistic hadronhadron collisions in the ultra-relativistic quantum molecular dynamics model," Journal of Physics G, vol. 25, no. 9, pp. 18591896, 1999.

[159] A. Majumder and M. van Leeuwen, "The theory and phenomenology of perturbative QCD based jet quenching," Progress in Particle and Nuclear Physics, vol. 66, no. 1, pp. 4192, 2011.

[160] A. Dainese, C. Loizides, and G. Paic, "Leading-particle suppression in high energy nucleus-nucleus collisions," European Physical Journal C, vol. 38, no. 4, pp. 461-474, 2005.

[161] I. Vitev and M. Gyulassy, "High-pT tomography of d+Au and $\mathrm{Au}+\mathrm{Au}$ at SPS, RHIC, and LHC," Physical Review Letters, vol. 89, no. 25, Article ID 252301, 4 pages, 2002.

[162] N. Armesto, A. Dainese, C. A. Salgado, and U. A. Wiedemann, "Testing the color charge and mass dependence of parton energy loss with heavy-to-light ratios at BNL RHIC and CERN LHC," Physical Review D, vol. 71, no. 5, Article ID 054027, 10 pages, 2005.

[163] T. Renk, H. Holopainen, R. Paatelainen, and K. J. Eskola, "Systematics of the charged-hadron PT spectrum and the nuclear suppression factor in heavy-ion collisions from $\sqrt{s_{N N}}=$ $200 \mathrm{GeV}$ to $\sqrt{s_{N N}}=2.76 \mathrm{TeV}$," Physical Review $C$, vol. 84 , no. 1 , Article ID 014906, 11 pages, 2011.

[164] S. Wicks, W. Horowitz, M. Djordjevic, and M. Gyulassy, "Elastic, inelastic, and path length fluctuations in jet tomography," Nuclear Physics A, vol. 784, no. 1-4, pp. 426-442, 2007.

[165] H. Zhang, J. F. Owens, E. Wang, and X. N. Wang, "Dihadron tomography of high-energy nuclear collisions in next-toleading order perturbative QCD," Physical Review Letters, vol. 98, no. 21, Article ID 212301, 4 pages, 2007.

[166] S. Chatrchyan, V. Khachatryan, A. M. Sirunyan et al., "Suppression of non-prompt $\mathrm{J} / \psi$, prompt $\mathrm{J} / \psi$, and $\Upsilon(1 \mathrm{~S})$ in $\mathrm{PbPb}$ collisions at $\sqrt{s_{N N}}=2.76 \mathrm{TeV}$,' Journal of High Energy Physics, vol. 1205, no. 5, article 63, 2012.

[167] E. Abbas and ALICE Collaboration, "J/ $\psi$ elliptic flow in $\mathrm{Pb}-\mathrm{Pb}$ collisions at $\sqrt{s_{N N}}=2.76 \mathrm{TeV}$," http://arxiv.org/abs/1303.5880.

[168] L. Adamczyk, J. K. Adkins, G. Agakishiev et al., "Measurement of $\mathrm{J} / \psi$ azimuthal anisotropy in $\mathrm{Au}+\mathrm{Au}$ collisions at $\sqrt{s_{N N}}=$ $200 \mathrm{GeV}$,' http://arxiv.org/abs/1212.3304.

[169] L. Adamczyk, J. K. Adkins, G. Agakishiev et al., "Jet-Hadron correlations in $\sqrt{s_{N N}}=200 \mathrm{GeV} \mathrm{Au}+\mathrm{Au}$ and $\mathrm{p}+\mathrm{p}$ collisions," http://arxiv.org/abs/1302.6184.

[170] S. Chatrchyan, V. Khachatryan, A.M. Sirunyan et al., "Observation of long-range, near-side angular correlations in $\mathrm{pPb}$ collisions at the LHC," Physics Letters B, vol. 718, no. 3, pp. 795814, 2013.

[171] L. Adamczyk, J. K. Adkins, G. Agakishiev et al., "Third harmonic flow of charged particles in Au+Au collisions at $\sqrt{s_{N N}}=$ 200 GeV,' Physical Review C, vol. 88, no. 1, Article ID 014904, 11 pages, 2013.
[172] G. Aad, T. Abajyan, B. Abbott et al., "Measurement with the ATLAS detector of multi-particle azimuthal correlations in $\mathrm{p}+\mathrm{Pb}$ collisions at $\sqrt{s_{N N}}=5.02 \mathrm{TeV}$," Physics Letters B, vol. 725, no. 1-3, pp. 60-78, 2013.

[173] S. S. Adler, S. Afanasiev, C. Aidala et al., "Detailed study of high-pT neutral pion suppression and azimuthal anisotropy in $\mathrm{Au}+\mathrm{Au}$ collisions at $\sqrt{s_{N N}}=200 \mathrm{GeV}$," Physical Review C, vol. 76, no. 3, Article ID 034904, 26 pages, 2007.

[174] A. Adare, S. Afanasiev, C. Aidala et al., "Quantitative constraints on the transport properties of hot partonic matter from semiinclusive single high transverse momentum pion suppression in $\mathrm{Au}+\mathrm{Au}$ collisions at $\sqrt{s_{N N}}=200 \mathrm{GeV}$," Physical Review C, vol. 77, no. 6, Article ID 064907, 12 pages, 2008.

[175] B. I. Abelev, M.M. Aggarwal, Z. Ahammed et al., "Energy dependence of $\pi \pm, \mathrm{p}$ and $\bar{p}$ transverse momentum spectra for $\mathrm{Au}+\mathrm{Au}$ collisions at $\sqrt{s_{N N}}=2.76$ and $200 \mathrm{GeV}$," Physics Letters $B$, vol. 655, no. 3-4, pp. 104-113, 2007. 

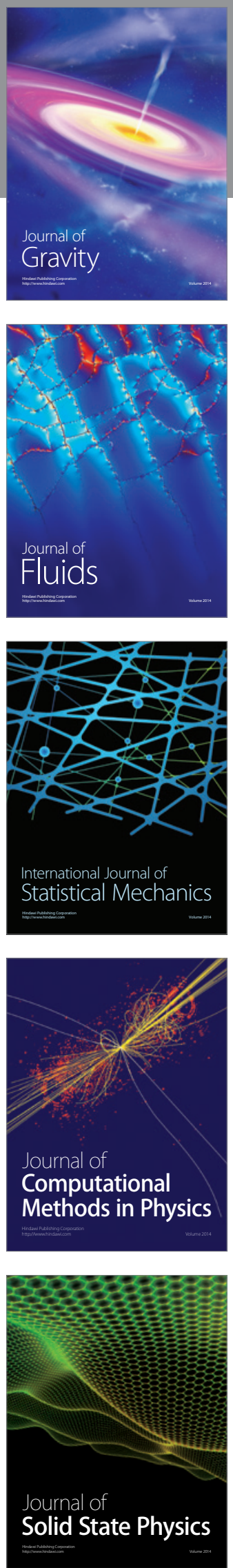

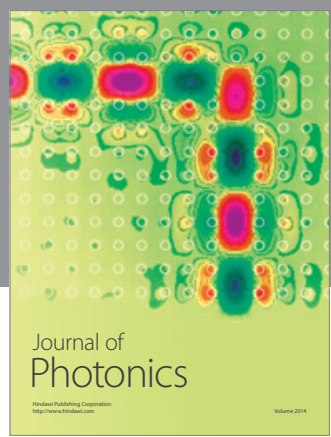

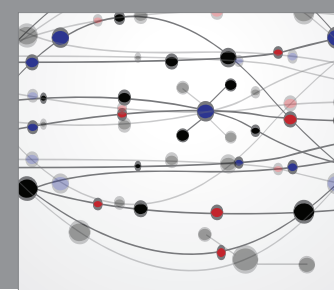

The Scientific World Journal

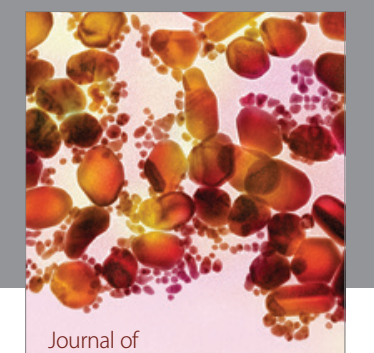

Soft Matter
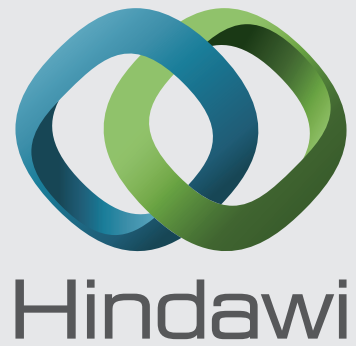

Submit your manuscripts at

http://www.hindawi.com
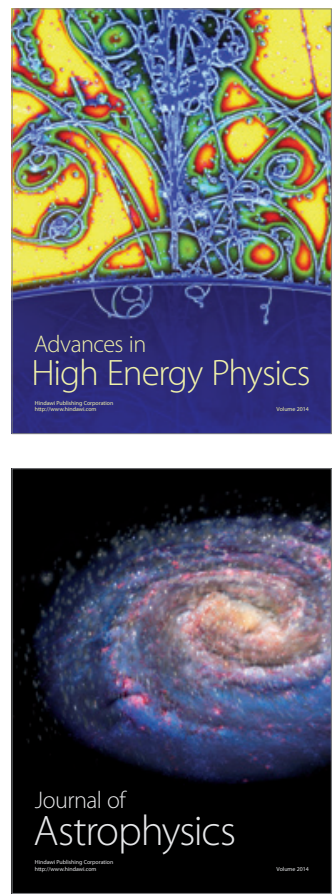
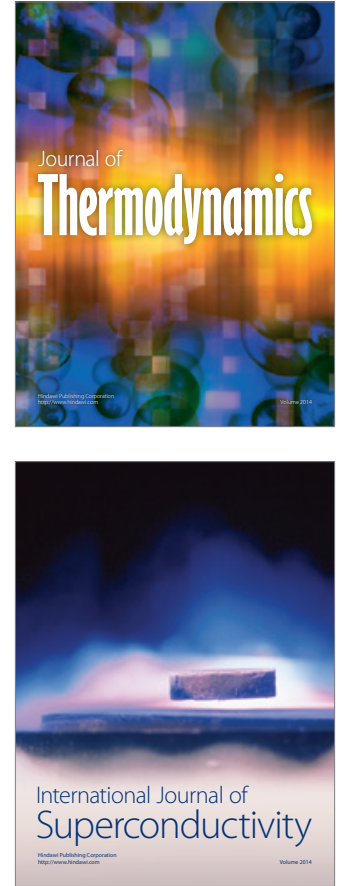
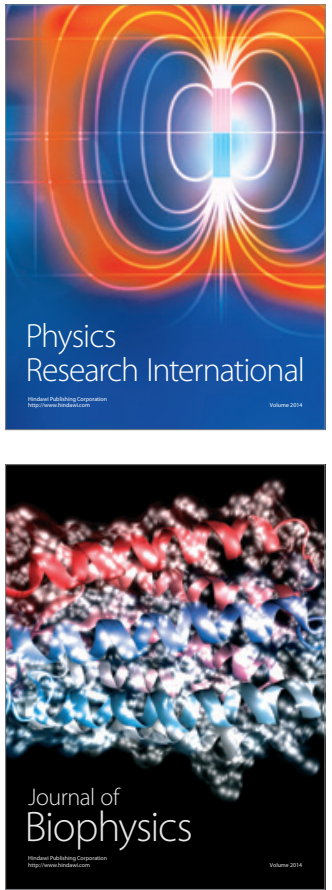
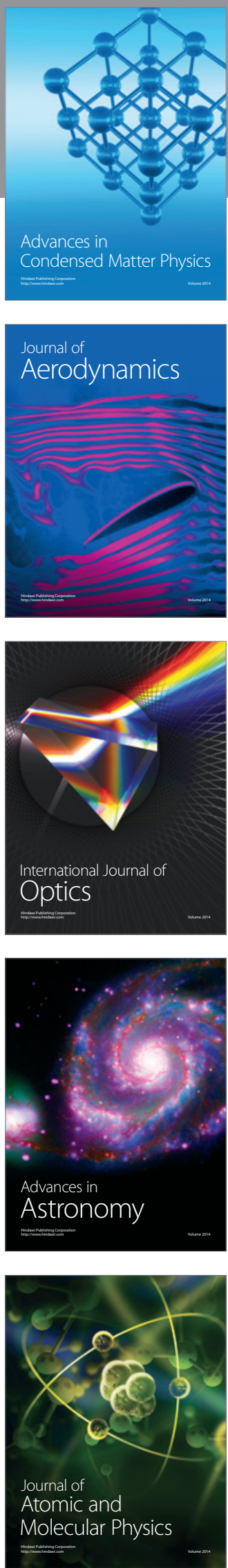